\title{
Kinematic Formula for Heterogeneous Gaussian Related Fields
}

\author{
Snigdha Panigrahi Jonathan Taylor Sreekar Vadlamani
}

\begin{abstract}
We provide a generalization of the Gaussian Kinematic Formula (GKF) in Taylor (2006) for multivariate, heterogeneous Gaussian-related fields. The fields under consideration, $f=F \circ y$, are non-Gaussian fields built out of smooth, independent Gaussian fields $y=\left(y_{1}, y_{2}, . ., y_{K}\right)$ with heterogeneity in distribution amongst the individual building blocks. Our motivation comes from potential applications in the analysis of Cosmological Data (CMB). Specifically, future CMB experiments will be focusing on polarization data, typically modeled as isotropic vector-valued Gaussian related fields with independent, but non-identically distributed Gaussian building blocks; this necessitates such a generalization. Extending results Taylor (2006) to these more general Gaussian relatives with distributional heterogeneity, we present a generalized Gaussian Kinematic Formula (GKF). The GKF in this paper decouples the expected Euler characteristic of excursion sets into Lipschitz Killing Curvatures (LKCs) of the underlying manifold and certain Gaussian Minkowski Functionals (GMFs). These GMFs arise from Gaussian volume expansions of ellipsoidal tubes as opposed to the usual tubes in the Euclidean volume of tube formulae. The GMFs form a main contribution of this work that identifies this tubular structure and a corresponding volume of tubes expansion in which the GMFs appear.
\end{abstract}

Keywords: Random Fields, Heterogeneous Fields, Gaussian processes, Euler Characteristic, Excursions, Kinematic Formula, Tube Formula.

\section{Smooth random fields and integral geometry}

Since the work of Adler (1981) and Worsley (1994), the study of smooth (usually Gaussian) random fields has exposed a very nice connection between properties of the excursion sets of the random fields and integral geometric properties of the parameter space of the field Worsley (1994). In more recent work, Taylor (2006) the integral geometric story has been extended to also include integral geometric properties of the marginal distribution (assumed constant) of the random field. This connection has been dubbed a Gaussian Kinematic Formula. In this work, we extend the GKF to a larger class of random fields, relaxing the assumption of identical distribution. Before stating our main result, we recall some classical quantities in integral geometry as well as earlier work in smooth random fields.

\subsection{Kinematic Formulae}

Perhaps the canonical example of integral geometric formulae are the kinematic fundamental formulae (KFF), having been applied in areas such as biology, mineralogy and metallurgy (see Santaló 
(2004) and references therein). Kinematic fundamental formulae are equalities establishing relationships between some averaged global geometric features of all possible intersections of two given bodies, and the global geometric quantities of individual bodies. In this sense, they can be viewed as generalizations of Buffon's needle problem.

In order to formulate the KFF, we dwell on the global geometric characteristics called the Lipschitz-Killing curvatures (LKCs) or intrinsic volumes, which are at the heart of such formulae. Given a $d$-dimensional smooth manifold $M$, LKCs are $(d+1)$ intrinsic, geometric functionals denoted as $\left\{\mathcal{L}_{k}(M)\right\}_{k=0}^{d}$ such that they satisfy following properties:

- each $\mathcal{L}_{k}$ for $k=0, \ldots, d$ is a finitely additive set functional;

- for any $\lambda>0$, and a nice set $A$, we have $\mathcal{L}_{k}(\lambda A)=\lambda^{k} \mathcal{L}_{k}(A)$, for all $k=0, \ldots, \operatorname{dim}(A)$;

- all $\mathcal{L}_{k}$ are rigid motion invariant i.e., for any nice set $A$, and any rigid motion $g$, writing $g A=\{g x: x \in A\}$ we have $\mathcal{L}_{k}(g A)=\mathcal{L}_{k}(A)$, for all $k=0, \ldots, \operatorname{dim}(A) ;$

- each $\mathcal{L}_{k}$ is continuous (we refer the reader to Klain and Rota (1997) for more details).

A simple example of encountering LKCs is the Steiner-Weyl tube formula (itself a special case of the KFF)

$$
\mathcal{H}_{k}(\operatorname{tube}(M, \epsilon))=\sum_{j=0}^{\operatorname{dim} M} \epsilon^{k-j} \operatorname{Vol}\left(B_{\mathbb{R}^{k}}(1)\right) \mathcal{L}_{j}(M),
$$

where $B_{\mathbb{R}^{k}}(1)$ is the unit ball in $\mathbb{R}^{k}$ and tube $(M, \epsilon)=\left\{y \in \mathbb{R}^{k}: \inf _{x \in M}\|x-y\| \leq \epsilon\right\}$. This gives the volume of an $\epsilon$-tubular neighborhood around a wide class of sets $M \subset \mathbb{R}^{k}$.

Equipped with the above definition/characterization of LKCs, we now state the most general Euclidean KFF, as it appears in Adler and Taylor (2007). Let $M_{1}$ and $M_{2}$ be two nice sets in $\mathbb{R}^{d}$, and let $\mathcal{G}_{d}$ be the group of rigid motions on $\mathbb{R}^{d}$, then

$$
\int_{\mathcal{G}_{d}} \mathcal{L}_{m}\left(M_{1} \cap g M_{2}\right) \nu(d g)=\sum_{j=0}^{d-m} \frac{s_{m+1} s_{d+1}}{s_{m+j+1} s_{d-j+1}} \mathcal{L}_{m+j}\left(M_{1}\right) \mathcal{L}_{d-j}\left(M_{2}\right)
$$

where $\nu$ is the normalised Haar measure on $\mathcal{G}_{d}$, and $s_{k}$ denotes the surface area of a unit ball in $\mathbb{R}^{k}$.

Remark 1.1. For a definition of nice sets we refer to Adler and Taylor (2007); Bröcker and Kuppe (2000).

KFFs have a rich history, and we refer the reader to Adler and Taylor (2007); Bröcker and Kuppe (2000); Klain and Rota (1997); Schneider and Weil (1992, 2008), and references therein, for an exhaustive account. All the available proofs of KFF are delicate, and rely heavily on various invariances available in the setup, like the invariance of Lebesgue measure under $\mathcal{G}_{d}$ plays a crucial role. A natural question then, one may ask, is if such integral formulae are exclusive only to Euclidean space with Lebesgue measure. 


\subsection{Excursion sets of Gaussian processes}

Interestingly, another problem which bears striking resemblance with Buffon's needle problem got many mathematicians interested. In order to exhibit the similarity, we can hypothesize the problem as having to sample a random path, instead of throwing a needle, and then counting the number of crossings of this random curve with a fixed line.

In 1940s, Kac (1943) and Rice (1945) solved this problem analytically with some basic regularity assumptions. Using a clever argument to count the number of crossings of a given function, Kac and Rice, working independently, obtained a compact expression for the mean number of crossings of a random algebraic function under some mild regularity conditions.

Revisiting (1), the case $m=0$ gives rise to the expected Euler-Poincaré characteristic (called Euler characteristic in the rest of this paper) denoted as $\chi($.$) . The expected Euler characteristic of$ excursion sets of smooth random fields $f$ on a $C^{3}$ domain $M$ defined as

$$
\mathbb{E}[\chi\{t \in M: f(t) \geq u\}],
$$

has been studied extensively in Adler (1981, 2000); Worsley (1994, 1995); Taylor and Adler (2003); Taylor (2006). The derivation of expected Euler characteristic for stationary Gaussian random fields dates back to Adler (1981), with generalizations to $\chi^{2}, \mathrm{~F}$ and t-fields and to higher dimensions in Worsley (1994, 1995). Adler (1981), and later, Taylor and Adler (2003), generalized the counting technique in Kac (1943); Rice (1945) to the multiparameter case. These papers set the stage for what is now called expectation metatheorem which can be viewed as quite general form of Kac-Rice formula (see Adler and Taylor (2007) for details). The expectation metatheorem can be stated as: let $n, k \geq 1$, and

$$
G=\left(G_{1}, \ldots, G_{n}\right) \text { and } H=\left(H_{1}, \ldots, H_{k}\right),
$$

be two $\mathbb{R}^{n}$ and $\mathbb{R}^{k}$ valued a.s. continuous random fields defined on an $n$-dimensional, parameter space $T \subset \mathbb{R}^{n}$ such that $T$ is smooth and compact. Let $U$ be an open subset of $\mathbb{R}^{k}$ such that the Hausdorff dimension of the boundary $\partial U=\bar{U} \backslash U$ is $(k-1)$, then writing

$$
N_{u}(G, H ; T, U)=\{x \in T: G(x)=u, \text { and } H(x) \in U\}
$$

under some regularity conditions (see (Adler and Taylor, 2007, Theorem 11.2.1)), we have

$$
\mathbb{E}\left(N_{u}(G, H ; T, U)\right)=\int_{T} \mathbb{E}\left\{|\operatorname{det} \nabla G| 1_{U}(H(x)) \mid G(x)=u\right\} p_{x}(u) d x,
$$

where $p_{x}$ is density of the random variable $G(x)$.

In Taylor and Adler (2003), the expected Euler characteristic for centered and unit variance, smooth Gaussian random fields $f$ on a smooth manifold $M$, based on the expectation meta theorem, 
was shown as a decoupling into LKCs of the manifold $M$ and coefficients that are products of Hermite polynomials with the standard Gaussian density. That is

$$
\mathbb{E}\left[\chi\left(M \cap f^{-1}[u, \infty)\right)\right]=\sum_{j=0}^{n} \mathcal{L}_{j}(M) \rho_{j}(u)
$$

with

$$
\rho_{j}(u)= \begin{cases}1-\Phi(u) & j=0 \\ \frac{1}{(2 \pi)^{(j+1) / 2}} H_{j-1}(u) \exp \left(-u^{2} / 2\right) & j \geq 1 .\end{cases}
$$

\subsection{Gaussian integral geometry and the GKF}

Taylor (2006) provided geometric meaning to the coefficients which appeared earlier in Adler (1981); Taylor and Adler (2003) via a Gaussian tube formula, and also extended the earlier calculations of Adler (1981); Worsley (1994) to a class of multivariate non-Gaussian random fields, which led to the formulation of Gaussian kinematic formula (GKF).

In the case of Gaussian random fields studied in Taylor and Adler (2003), the EC densities $\rho_{j}(u)$ are seen to match up to a factor of $(2 \pi)^{-j / 2}$ with the coefficients

$$
\mathcal{M}_{j}^{\gamma^{\mathbb{R}^{1}}}([u, \infty))=(2 \pi)^{-1 / 2} H_{j-1}(u) \exp \left(-u^{2} / 2\right)
$$

arising in a Gaussian tubular volume expansion $\gamma^{\mathbb{R}^{1}}(\operatorname{tube}([u, \infty), \epsilon))=\gamma^{\mathbb{R}^{1}}([u-\epsilon, \infty))$.

The GKF formula, more generally, can be stated as a decoupling of the mean LKCs of excursion sets into LKCs of $M$ and GMFs that are seen in the tube formula. Suppose $f=F \circ y$ whose components are smooth, independent and marginally stationary with marginal law $N(0,1)$. Then,

$$
\mathbb{E}\left[\mathcal{L}_{m}\left(M \cap f^{-1}[u, \infty)\right)\right]=\sum_{j=0}^{\operatorname{dim} M-m}\left[\begin{array}{c}
m+j \\
j
\end{array}\right](2 \pi)^{-j / 2} \mathcal{L}_{m+j}(M) \mathcal{M}_{j}^{\gamma_{\mathbb{R}}^{K}}\left(F^{-1}[u, \infty)\right)
$$

with $\left[\begin{array}{c}m+j \\ j\end{array}\right]=\frac{(m+j) ! \nu_{n}}{m ! j ! \nu_{m} \nu_{j}}$ and $\nu_{j}=\frac{\pi^{j / 2}}{\Gamma(n / 2+1)}$ the volume of a unit ball in $\mathbb{R}^{n}$.

The above, re-derived in Taylor et al. (2009) can be viewed as recasting (1) in the form of a KFF over Gaussian function space. The Gaussian Minkowski Functionals in Taylor et al. (2009) are defined implicitly in a generalization of the Steiner-Weyl formula

$$
\gamma_{\mathbb{R}^{K}}(\operatorname{tube}(\mathcal{K}, \epsilon))=\gamma_{\mathbb{R}^{K}}\left(\mathcal{K} \oplus B_{\mathbb{R}^{K}}(\epsilon)\right)=\sum_{j \geq 0} \frac{\epsilon^{j}}{j !} \mathcal{M}_{j}^{\gamma_{\mathbb{R}}^{K}}(\mathcal{K}) .
$$




\subsection{Extension of GKF}

Formulae for the expected Euler characteristic of a smooth Gaussian field has already found many important applications in the analysis of cosmological data (CMB), see Fantaye et al. (2015), Collaboration et al. (2014), Ade et al. (2015) for more details on this. Isotropic vector-valued Gaussian related fields with independent, but non-identically distributed Gaussian building blocks can arise in modeling of polarization data in CMB experiments, necessitating a generalization of GKF in Taylor (2006) to multivariate, heterogeneous, Gaussian related random fields. Motivated by applications in CMB experiments, our goal in this paper is to derive a GKF for heterogenous, Gaussian relatives $f=F \circ y$, constructed out of Gaussian fields $y=\left(y_{1}, y_{2}, \ldots, y_{K}\right)$ with distributional heterogenity in individual components. That is, the component Gaussian fields are marginally stationary and independent, but non-identically distributed. Specifically, the building blocks are non-identical in distribution in the following sense: the gradient field $\nabla y$ has separable covariance structure

$$
\operatorname{diag}\left(\lambda_{1}, \ldots, \lambda_{K}\right) \otimes I \stackrel{\text { def }}{=} D \otimes I,
$$

and induces conformal Riemannian metrics (with constant conformal factor) on the manifold $M$. As such our prototypical model in this work has $M=S\left(\mathbb{R}^{3}\right)$ and each $y_{i}$ is isotropic on the sphere with possibly different spectral measures.

The main theorem of the paper derives a $(\mathrm{GKF})$ for the expected Euler characteristic of the excursion sets of such fields $f$, yielding the result

$$
\mathbb{E}\left[\chi\left(M \cap f^{-1}[u, \infty)\right]=\mathbb{E}\left[\chi\left(M \cap y^{-1} \mathcal{K}\right)\right]=\sum_{j=0}^{n}(2 \pi)^{-j / 2} \mathcal{L}_{j}(M) \mathcal{M}_{j}^{\gamma_{\mathbb{R}}^{K}, D}(\mathcal{K}),\right.
$$

with $\mathcal{K}=F^{-1}[u, \infty), \mathcal{L}_{j}(M)$ the LKCs of $M$, and $\mathcal{M}_{j}^{\gamma_{\mathrm{R}}^{K}, D}(\mathcal{K})$ the Gaussian Minkowski functionals.

In comparing (3) to (4) the reader will notice that we have introduced a parameter $D$ to the Gaussian Minkowski functionals in (4). Define an ellipsoidal tube with as

$$
T^{D}(\mathcal{K}, \epsilon)=\mathcal{K} \oplus B_{D, \mathbb{R}^{K}}(\epsilon),
$$

with

$$
B_{D, \mathbb{R}^{K}}(\epsilon)=\left\{w \in \mathbb{R}^{K}: w^{T} D^{-1} w \leq \epsilon^{2}\right\}
$$

recalling that $D=\operatorname{diag}\left(\lambda_{1}, \ldots, \lambda_{K}\right)$.

The GMFs above are implicitly defined as terms in a Taylor series expansion for the Gaussian volume of $T^{D}(\mathcal{K}, \epsilon)$ in terms of integrals on the boundary of $\mathcal{K}$ given by

$$
\gamma_{\mathbb{R}^{K}}\left(T^{D}(\mathcal{K}, \epsilon)\right)=\gamma_{\mathbb{R}^{K}}(\mathcal{K})+\sum_{l=1}^{\infty} \frac{\epsilon^{l}}{l !} \mathcal{M}_{l}^{\gamma_{\mathbb{R}^{K}}, D}(\mathcal{K}) .
$$


Note then that the original GMFs in (3) simply correspond to the case $D=I$.

\subsection{Outline of the paper}

The main result is stated formally in Section 2, and is established as follows. We show in Section 4 that the expected Euler Characteristic for the Gaussian related fields under consideration can be expanded in terms of EC densities, computed as integrals with respect to standard Gaussian measure and Lipschitz Killing curvatures (LKC). The LKCs are derived from the Riemannian curvature induced by the base spatial metric $g$ on $M$. Section 5 is devoted to obtaining explicit integral representations of EC densities by carefully modifying the tools developed in Taylor (2006) to be adapted to our setting. The result follows with the observation that the coefficients in volume expansions of the ellipsoidal tubes considered in Section 3 and the integral representation of $\left\{\tilde{\rho}_{j}(F, u) ; j=1,2, . ., n\right\}$ in Section 5 match up to a factor of $(2 \pi)^{-j / 2}$, leading to an analogous GKF for heterogeneous Gaussian related fields. Finally, in Section 6 we conclude with an application of our results to the study of cosmic microwave background radiation data.

\section{GKF for heterogeneous Gaussian fields}

In this section, we formally state a generalization of the GKF in Taylor (2006) which somewhat relaxes distributional assumptions. The essence of such a result lies in the decoupling of spatial information and distributional information of the random field. We begin with formally describing our assumptions on the $\mathbb{R}^{K}$ valued field $y$.

\subsection{Set up and assumptions}

We describe the heterogeneous Gaussian related fields $f=F \circ y$ under consideration in the paper by listing a set of assumptions on the individual Gaussian building blocks $y$ and the function $F$.

The Gaussian building blocks $y=\left(y_{1}, . ., y_{K}\right)$ in particular are assumed to satisfy the following assumptions:

(A) Marginal Stationarity and Independence: $\left(y_{1}, . ., y_{K}\right)$ are individually real-valued, mean 0 , unit variance, independent random fields on manifold $M$.

(B) Separability of gradient field: We assume a separable structure for the covariance of the gradient field

$$
\operatorname{Cov}(\nabla y)=D \otimes I
$$

where $D=\operatorname{diag}\left(\lambda_{1}, \ldots, \lambda_{K}\right)$. Here $D$ represents the covariance amongst the random fields, while $I$ denotes the spatial covariance. 
(C) Metric Conformity: Each $y_{k}$ induces a metric $g_{k}$ on the manifold $M$ such that

$$
g_{i, j}^{k}=g^{k}\left(E_{i}, E_{j}\right)=\lambda_{k} g\left(E_{i}, E_{j}\right),
$$

where $\left\{E_{i}\right\}$ being an orthonormal frame field with respect to base spatial metric $g$ and $\lambda_{k}$ being the second spectral moment of the field $y_{k}$.

(D) Regularity: The tuple $Y_{k}=\left(y_{k}(t), \nabla y_{k}(t), \nabla^{2} y_{k}(t)\right)$ should satisfy:

$$
\mathbb{P}\left(\sup _{u \in \mathcal{B}(t, h)}\left\|Y_{k}(t)-Y_{k}(u)\right\|_{2}>\epsilon\right)=o\left(h^{n}\right)
$$

for any $\epsilon>0$, for the metric \|\|$_{2}$ defined as :

$$
\left\|Y_{k}(t)\right\|_{2}=\left|y_{k}(t)\right|+\left\|\nabla y_{k}(t)\right\|_{\mathbb{R}^{n}}+\left\|\nabla^{2} y_{k}(t)\right\|_{\otimes^{2} \mathbb{R}^{n}}
$$

for ball $\mathcal{B}(t, h)$ around $t$ with radius $h$.

Remark 2.1. Under the assumption of marginal stationarity, we have independence of the gradient field from the field and hessian evaluated at a point $t$, that is

$$
\nabla y_{k}(t) \perp\left(y_{k}, \nabla^{2} y_{k}\right)(t) \text { for } k \in\{1,2, \ldots, K\}
$$

Separability along with marginal stationarity ensures that

$$
\operatorname{Cov}\left(\frac{\partial y_{k}(t)}{\partial t_{i}}, \frac{\partial y_{k}(t)}{\partial t_{j}}\right)=-\operatorname{Cov}\left(y_{k}(t), \frac{\partial^{2} y_{k}(t)}{\partial t_{i} \partial t_{j}}\right)=\lambda_{k} \delta_{i, j} I \text { for } k \in\{1,2, \ldots, K\} .
$$

Remark 2.2. We can choose to replace assumptions $(\mathbf{A})$ and $(\mathbf{C})$ with the stronger assumption of isotropic, centered and unit variance, independent Gaussian random fields. Isotropy would imply metric conformity, as desired in $\mathbf{( C )}$.

Remark 2.3. We can in fact assume that in $\mathbf{( B )}$ that

$$
\operatorname{Cov}(\nabla y)=D \otimes \nu I \text { for some } \nu \in \mathbb{R}^{+},
$$

with a scaled spatial covariance matrix $\nu$ I. In such a case, the spatial scale parameter $\nu$ shows up in our GKF in 2.5 as scaled LKCs with a scaling factor of $\nu^{j / 2}$ for $\mathcal{L}_{j}$. We elaborate on this remark in Section 4 in Remark 4.7. In all our of our calculations, we assume $\nu=1$.

Note that, we enforce distributional heterogenity amongst the building blocks

$$
\left\{y_{i}, \quad i \in 1,2, . ., K\right\}
$$


by considering a KP separable covariance structure for the gradient fields $\nabla y$, such that metrics induced by them are conformal in nature. We emphasize that all the geometric calculations are with respect to the base spatial metric $g$.

We need some assumptions on the function $F$, which when composed with the above nonidentically distributed but independent Gaussian fields $y$, yields heterogeneous Gaussian relatives $f$, our fields of interest. We assume that $F \in \mathcal{C}^{2}\left(\mathbb{R}^{K}\right)$ is real valued and satisfies for some $\epsilon>0$ :

(1) $\|\nabla F\|$ is bounded on both sides on $F^{-1}(u-\epsilon, u+\epsilon)$

(2) $\nabla F$ is Lipschitz on $F^{-1}(u-\epsilon, u+\epsilon)$

(3) Functions $\tilde{\mathcal{C}}_{F}$, discussed in $(30)$ are continuous in $(u-\epsilon, u+\epsilon)$

(4) $\lim _{\varepsilon \rightarrow 0} \frac{1}{2 \varepsilon} \mathbb{E}\left[1_{\{|F(y)-u|<\varepsilon\}}\left|H_{n-1-l}(-\langle y, D F(y)\rangle)\left\|D^{2} \nabla^{2} F\right\|_{\otimes^{2 l} \mathbb{R}^{K}}\right|\right]<\infty$, for all $n, l$.

Finally, we assume that the domain set $\mathcal{K}=F^{-1}[u, \infty)$ is smooth and convex, with shape operator of $\partial \mathcal{K}$ bounded and critical radius of $\mathcal{K}$ positive.

Remark 2.4. When the set $\mathcal{K}$ is non-smooth, but locally convex, then similar calculations hold, though we will have to be careful about breaking up calculations on different pieces.

\subsection{Main result}

With $y, F$ and domain set $\mathcal{K}$ satisfying assumptions listed in Section 2.1 above, we are ready to state the GKF theorem for the heterogeneous Gaussian relatives with independent, marginally stationary components

$$
\left\{y_{i}, i=1,2, \ldots, K\right\}
$$

having separable structure for the gradient field and inducing conformal metrics. Even more strictly, we can consider isotropic Gaussian components with separability for corresponding gradient fields. We outline the proof briefly in this section, proving the details of the results involved in the GKF in the following sections.

Theorem 2.5. GKF generalized to heterogeneous Gaussian Related Fields: For $F \in \mathcal{C}\left(\mathbb{R}^{K}\right)$ satisfying (1), (2), (3) and (4) in the assumptions for $F, \mathcal{K}=F^{-1}[u, \infty)$ convex and smooth, and Gaussian random fields $y=\left(y_{1}, . ., y_{K}\right)$, satisfying assumptions $(\mathbf{A}),(\mathbf{B}),(\mathbf{C})$ and $(\mathbf{D})$ on $M$, the kinematic formula for $f=F \circ y$ can be expressed as

$$
\mathbb{E}\left[\chi\left(M \cap y^{-1} \mathcal{K}\right)\right]=\sum_{j=0}^{n}(2 \pi)^{-j / 2} \mathcal{L}_{j}(M) \mathcal{M}_{j}^{\gamma_{\mathbb{R}}^{K}, D}(\mathcal{K})
$$

where the coefficients in the above expansion decouple into $\left\{\mathcal{L}_{j}(M)\right\}_{0 \leq j \leq n}$ (LKCs), computed with respect to spatial metric $g$ and $\left\{\mathcal{M}_{j}^{\gamma_{\mathbb{R}}^{K}, D}(\mathcal{K})\right\}_{0 \leq j \leq n}$ (GMFs), arising as coefficients in the Taylor series expansion of Gaussian volumes of ellipsoidal tubes as in (3) 
Proof. The expected Euler Characteristic is derived in terms of the LKCs on $M$ and the EC density functionals in Section 4 as

$$
\mathbb{E}\left[\chi\left(M \cap y^{-1} \mathcal{K}\right)\right]=\sum_{j=0}^{n} \mathcal{L}_{j}(M) \rho_{j}(F, u) .
$$

Independently, the Gaussian volume expansion of ellipsoidal tubes is computed as an expansion in GMFs, denoted as $\left\{\mathcal{M}_{j}^{\gamma_{\mathrm{R}}^{K}, D}(\mathcal{K})\right\}_{0 \leq j \leq n}$ in Section 3. Having done the computations above, it is a matter of verifying that the coefficients $\mathcal{M}_{j}^{\gamma_{\mathbb{R}}^{K}, D}(\mathcal{K})$ in Theorem 3.5, followed with the derivation of EC densities $\tilde{\rho}_{n}(F, u)$ for fields $f$ in 5.2 match up to constants.

Realizing that $\partial \mathcal{K}=F^{-1} u$ and $\eta_{y}$, the outward unit normal at $y \in \partial \mathcal{K}$ in 3.5, identifies with $\nabla F(y)$, we see that

$$
\left(\frac{\left\|D \eta_{y}\right\|}{\left\|D^{1 / 2} \eta_{y}\right\|}\right)^{l-1-m} H_{l-1-m}\left(\frac{\left\langle y, D \eta_{y}\right\rangle}{\left\|D \nabla \eta_{y}\right\|}\right)
$$

in (15), coming from a Taylor series expansion of the standard Gaussian density function matches with

$$
\left(\frac{\|D \nabla F(y)\|}{\left\|D^{1 / 2} \nabla F(y)\right\|}\right)^{l-1-m} H_{l-1-m}\left(\frac{\langle D \nabla F(y), y\rangle}{\|D \nabla F(y)\|}\right),
$$

in $(29)$.

The other term in the $\epsilon$ neighborhood expansion of the ellipsoidal tube, denoted as

$$
\mathcal{M}_{m+1}^{*}(\mathcal{K}, d x)
$$

derived from the Jacobian of an appropriate transformation, parametrizing the boundary of the ellipsoidal tube, in 3.3 , matches with

$$
\operatorname{Tr}^{\nabla F^{\perp}}\left(D \nabla^{2} F_{\mid \nabla F^{\perp}} /\left\|D^{1 / 2} \nabla F(y)\right\|\right)^{m} \mathcal{H}_{K-1}(d x)
$$

in (29). The two computations in (15) and (29) help us conclude that the EC densities indeed, agree up to constants with the coefficients of volumes of ellipsoidal tubes $T^{D}(\mathcal{K}, \epsilon)$, yielding

$$
\tilde{\rho}_{j}(F, u)=\frac{1}{(2 \pi)^{j / 2}} \mathcal{M}_{j}^{\gamma_{\mathbb{R}}^{K}, D}(\mathcal{K})
$$

and thus, follows the Kinematic formula.

As we go through the subsequent sections, all the claims made in the proof above become clear. 


\section{Gaussian volumes of ellipsoidal tubes}

In this section, we devote our attention to tubular expansions of certain geometric objects, which we refer to as "ellipsoidal tubes", computed with respect to the standard Gaussian measure. Computations for volumes of regular tube neighborhoods around $M$ can be seen as early as in Weyl (1939) for an embedded manifold $M \subset \mathbb{R}^{n}$ and are credited to Steiner for compact, convex $M$. These volume expansions have also found place in Gray (1990); Schneider (2013) later. For us, the geometric objects whose expansions lead to a GKF for heterogeneous, Gaussian related fields are no longer the regular tubes. The non-homogeneity in distribution of component Gaussian fields leads to a different geometry, which we call ellipsoidal tubes and define them below.

In particular, we compute the Gaussian volume of an ellipsoidal tube around $\mathcal{K}$ where

$$
\mathcal{K}=F^{-1}[u, \infty) \in \mathbb{R}^{K},
$$

is assumed to be smooth and convex.

For a positive definite $\Theta \in \mathbb{R}^{K \times K}$, we begin with the observation that the boundary of the set $\mathcal{K} \oplus B_{\Theta, \mathbb{R}^{K}}(\epsilon)$ for a convex, smooth set $\mathcal{K}$ can be parametrized by the map

$$
\left(x, \eta_{x}\right) \rightarrow x+\epsilon \frac{\Theta \eta_{x}}{\left\|\eta_{x}\right\|_{\Theta}}
$$

where $\eta_{x}$ is the outward unit normal at $x$ on $\partial \mathcal{K}$, and a $\Theta$-norm is defined as:

$$
\|x\|_{\Theta}^{2}=x^{T} \Theta x
$$

Lemma 3.1. For $\mathcal{K}$ convex and $\Theta \in \mathbb{R}^{K \times K}>0$, the map

$$
S(N(\mathcal{K})) \ni\left(z, \eta_{z}\right) \mapsto z+\epsilon \frac{\Theta \eta_{z}}{\left\|\eta_{z}\right\|_{\Theta}}
$$

parameterizes $\partial\left(\mathcal{K} \oplus B_{\Theta, \mathbb{R}^{K}}(\epsilon)\right)$, where

$$
\|z-y\|_{\Theta^{-1}}^{2}=(z-y)^{T} \Theta^{-1}(z-y) .
$$

Proof. For $y \in \mathbb{R}^{K}$, consider the problem

$$
\underset{z \in \mathcal{K}}{\operatorname{minimize}}\|z-y\|_{\Theta^{-1}}^{2}
$$

where

$$
\|z-y\|_{\Theta^{-1}}^{2}=(z-y)^{T} \Theta^{-1}(z-y)
$$


as defined in the statement of the Lemma. For $y \in \mathcal{K}^{c}$, solving the above problem yields a pair

$$
(\hat{z}(y), y-\hat{z}(y)) \in N(\partial \mathcal{K})
$$

satisfying the KKT conditions

$$
\Theta^{-1}(\hat{z}(y)-y)=-c(y) \cdot(y-\hat{z}(y))
$$

for $c(y) \geq 0$ and $y-\hat{z}(y)$ is in the normal cone to $\mathcal{K}$ at $\hat{z}(y)$.

Hence, solving this problem determines a map $h: \mathcal{K}^{c} \rightarrow N(\partial \mathcal{K})$. This map has an inverse $\bar{h}$ defined

$$
\left(z, \eta_{z}\right) \mapsto z+\Theta \eta_{z}
$$

with $\Theta=Q^{-1}$. That is,

$$
\Theta^{-1}\left(z-\bar{h}\left(z, \eta_{z}\right)\right)=\eta_{z}
$$

and hence

$$
z=\underset{w \in \mathcal{K}}{\operatorname{argmin}}\left\|w-\bar{h}\left(z, \eta_{z}\right)\right\|_{\Theta^{-1}}^{2} .
$$

(At the risk of being a little pedantic we are identifying the matrix $\Theta$ with a linear mapping $T_{y} \mathbb{R}^{K} \rightarrow T_{y} \mathbb{R}^{K}$ assuming that the basis of this mapping in the standard basis is $\Theta$. It therefore makes sense to write $\Theta E$ and $\Theta^{-1} E$ for vector fields on $\mathbb{R}^{K}$ or $\partial \mathcal{K}$.)

Each $\left(z, \eta_{z}\right)$ has a value associated to it: $V_{\mathcal{K}}\left(\bar{h}\left(z, \eta_{z}\right)\right)$ where

$$
V_{\mathcal{K}}(y)=\inf _{z \in \mathcal{K}}\|z-y\|_{\Theta^{-1}}^{2}
$$

We note here that

$$
\left\{y: V_{\mathcal{K}}(y) \leq \epsilon^{2}\right\}=\mathcal{K} \oplus B_{\Theta, \mathbb{R}^{K}}(\epsilon) .
$$

We parameterize the set

$$
\left\{y: V_{\mathcal{K}}(y)=\epsilon^{2}\right\}=\partial\left(\mathcal{K} \oplus B_{\Theta, \mathbb{R}^{K}}(\epsilon)\right)
$$

by constructing a map from $S(N(\mathcal{K})$ ), the unit normal vectors of $\mathcal{K}$ to this set. Clearly,

$$
V_{\mathcal{K}}\left(\bar{h}\left(z, c \eta_{z}\right)\right)=c^{2}\left\|\eta_{z}\right\|_{\Theta}^{2} \quad \forall\left(z, \eta_{z}\right) \in S(N(\mathcal{K})), c>0
$$

hence choosing

$$
c\left(z, \eta_{z}\right)=\frac{\epsilon}{\left\|\eta_{z}\right\|_{\Theta}}
$$

yields the desired parameterization. 
Corollary 3.2. Taking $\Theta=D=\operatorname{diag}\left(\lambda_{1}, \ldots, \lambda_{K}\right)$ yields the map which parametrizes the boundary of $T^{D}(\mathcal{K}, \epsilon)=\mathcal{K} \oplus B_{D, \mathbb{R}^{K}}(\epsilon)$ where

$$
\mathcal{K}=F^{-1}[u, \infty) \in \mathbb{R}^{K},
$$

which we assume to be convex and smooth and $B_{D, \mathbb{R}^{K}}(\epsilon)$ is the ellipsoid given by the set

$$
\left\{x \in \mathbb{R}^{K}: x^{T} D^{-1} x \leq \epsilon^{2}\right\} .
$$

With the above parametrization of the surface of $\mathcal{K} \oplus B_{D, \mathbb{R}^{K}}(\epsilon)$, the next lemma evaluates the Jacobian of the transformation

$$
\left(x, \eta_{x}\right) \rightarrow x+\epsilon \frac{D \eta_{x}}{\left\|\eta_{x}\right\|_{D}}
$$

used to project a small patch $\mathcal{A}_{\epsilon}$ on $\partial\left(\mathcal{K} \oplus B_{D, \mathbb{R}^{K}}(\epsilon)\right)$ onto patch $\mathcal{A}$ on $\partial \mathcal{K}$.

Lemma 3.3. Again, under the assumption of $\mathcal{K}$ being smooth, $\Theta \in \mathbb{R}^{K \times K}>0$ and

$$
\eta_{z}=-\nabla F(z) /\|\nabla F(z)\|_{2}
$$

taken as the unique outward pointing normal vector field which can be extended to a smooth vector field on a neighborhood of any patch on $\partial \mathcal{K}$, the change of basis matrix for the transformation

$$
\left(z, \eta_{z}\right) \mapsto z+\epsilon \frac{\Theta \eta_{z}}{\left\|\eta_{z}\right\|_{\Theta}}
$$

is given by

$$
\operatorname{det}\left(I_{K-1}+\epsilon \cdot A(z)\right)=\sum_{j=0}^{K-1} \epsilon^{j} \operatorname{detr}_{j}(A(z))
$$

where,

$$
A(z)_{i j}=\left.\frac{1}{\|\nabla F(z)\|_{\Theta}}\left\langle\nabla_{E_{i}} \Theta \nabla F, E_{j}\right\rangle_{\Theta^{-1}}\right|_{z}
$$

Proof. Let's look at the derivative of a linear projection of our map. Ignoring the term $z$ define

$$
\varsigma(z)=\frac{\epsilon \Theta \eta_{z}}{\left\|\eta_{z}\right\|_{\Theta}}
$$

A straightforward calculation shows that for any vector field $X$ on $\mathbb{R}^{K}$

$$
\begin{aligned}
\left\langle\nabla_{X} \varsigma, v\right\rangle_{I} & =\epsilon\left\langle\frac{\Theta^{1 / 2} \nabla_{X} \eta}{\|\eta\|_{\Theta}},\left(I-\frac{\Theta^{1 / 2} \eta \eta^{T} \Theta^{1 / 2}}{\|\eta\|_{\Theta}^{2}}\right) \Theta^{1 / 2} v\right\rangle_{I} \\
& =\epsilon\left\langle\frac{\nabla_{X} \eta}{\|\eta\|_{\Theta}},\left(\Theta-\frac{\Theta \eta \eta^{T} \Theta}{\|\eta\|_{\Theta}^{2}}\right) v\right\rangle_{I}
\end{aligned}
$$


with $\langle,\rangle_{I}$ the usual Euclidean inner product, $\Theta^{1 / 2}$ the symmetric square root of $\Theta$ (we could take non-symmetric square-roots if we had to, but it doesn't matter - everything below makes sense without square roots), and $\nabla$ the usual Euclidean Levi-Civita connection (i.e. standard differentiation of vector fields). Letting $Q=\Theta^{-1}$, define

$$
\langle X, Y\rangle_{Q}=\langle X, Q Y\rangle_{I}
$$

Now, choose a frame $\left\{E_{1}, \ldots, E_{K}\right\}$ such that

$$
\left\langle E_{i}, E_{j}\right\rangle_{Q}=\delta_{i j}, \quad 1 \leq i, j \leq K
$$

with

$$
E_{K}=\frac{\Theta \eta}{\|\eta\|_{\Theta}}
$$

Noting that $\varsigma(z)=\epsilon E_{K}(z)$ it suffices to differentiate $E_{K}$ to compute the change of measure term. Note that, for $1 \leq i \leq K-1,1 \leq j \leq K$ :

$$
\begin{aligned}
0 & =E_{i}\left\langle E_{K}, E_{j}\right\rangle_{Q} \\
& =E_{i}\left\langle E_{K}, Q E_{j}\right\rangle_{I} \\
& =\left\langle E_{K}, Q \nabla_{E_{i}} E_{j}\right\rangle_{I}+\left\langle\nabla_{E_{i}} E_{K}, Q E_{j}\right\rangle_{I} \\
& =\frac{1}{\|\eta\|_{\Theta}}\left\langle\eta, \nabla_{E_{i}} E_{j}\right\rangle_{I}+\left\langle\nabla_{E_{i}} E_{K}, E_{j}\right\rangle_{Q} \\
& =-\frac{1}{\|\eta\|_{\Theta}}\left\langle\nabla_{E_{i}} \eta, E_{j}\right\rangle_{I}+\left\langle\nabla_{E_{i}} E_{K}, E_{j}\right\rangle_{Q} .
\end{aligned}
$$

The last display uses the assumption that $\left\langle E_{i}, \eta\right\rangle_{I}=0$ for $1 \leq i \leq K-1$ which is implied by our choice of frame $\left\{E_{1}, \ldots, E_{K}\right\}$.

Our calculation (11) shows that

$$
\begin{aligned}
\left\langle\nabla_{E_{i}} E_{K}, E_{K}\right\rangle_{Q} & =\left\langle\nabla_{E_{i}} E_{K}, \frac{\eta}{\|\eta\|_{\Theta}}\right\rangle_{I} \\
& =\frac{1}{\|\eta\|_{\Theta}}\left\langle\nabla_{E_{i}} E_{K}, \eta\right\rangle \\
& =0 .
\end{aligned}
$$


Therefore,

$$
\begin{aligned}
\nabla_{E_{i}} E_{K} & =\sum_{j=1}^{K}\left\langle\nabla_{E_{i}} E_{K}, E_{j}\right\rangle_{Q} E_{j} \\
& =-\sum_{j=1}^{K-1}\left\langle\frac{\eta}{\|\eta\|_{\Theta}}, \nabla_{E_{i}} E_{j}\right\rangle_{I} E_{j} \\
& =\frac{1}{\|\eta\|_{\Theta}} \sum_{j=1}^{K-1}\left\langle\nabla_{E_{i}} \eta, E_{j}\right\rangle_{I} E_{j}
\end{aligned}
$$

In terms of our frame, this means the change of basis matrix for the transformation is therefore

$$
\operatorname{det}\left(I_{K-1}+\epsilon \cdot A(z)\right)
$$

where

$$
\begin{aligned}
A(z)_{i j} & =\left.\frac{1}{\left\|\eta_{z}\right\|_{\Theta}}\left\langle\nabla_{E_{i}} \eta, E_{j}\right\rangle_{I}\right|_{z} \\
& =\left.\frac{1}{\left\|\eta_{z}\right\|_{\Theta}}\left\langle\nabla_{E_{i}} \Theta \eta, E_{j}\right\rangle_{Q}\right|_{z} \\
& =\left.\frac{1}{\|\nabla F(z)\|_{\Theta}}\left\langle\nabla_{E_{i}} \Theta \nabla F, E_{j}\right\rangle_{Q}\right|_{z}
\end{aligned}
$$

Finally, the determinant is a polynomial in $\epsilon$

$$
\operatorname{det}\left(I_{K-1}+\epsilon \cdot A(z)\right)=\sum_{j=0}^{K-1} \epsilon^{j} \operatorname{detr}_{j}(A(z))
$$

To calculate the volume of $T^{D}(\mathcal{K}, \epsilon)$, we compute the surface area of an infinitesimally small patch $\mathcal{A}_{\epsilon}$ on the surface of $T^{D}(\mathcal{K}, \epsilon)$ by using the map in 3.1 and the Jacobian in 3.3 to project back to $\partial \mathcal{K}$. Integrating over $\partial \mathcal{K}$ and over $[0, \epsilon]$, we get an expansion for the volume for the ellipsoidal tube. Based on 3.1 and 3.3, the next corollary gives the surface measure of a small patch $\mathcal{A}_{\epsilon}$ on the surface of the ellipsoidal tube $\partial\left(\mathcal{K} \oplus B_{\Theta, \mathbb{R}^{K}}(\epsilon)\right)$.

Corollary 3.4. The surface measure of a patch $\mathcal{A}_{\epsilon}$ on the surface of $\partial\left(\mathcal{K} \oplus B_{\Theta, \mathbb{R}^{K}}(\epsilon)\right)$ is given by

$$
\mathcal{H}_{K-1}\left(\mathcal{A}_{\epsilon}\right)=\sum_{j=1}^{K} \frac{\epsilon^{j-1}}{(j-1) !} \mathcal{M}_{j}^{*}(\mathcal{K}, \mathcal{A})
$$

where

$$
\mathcal{M}_{j}^{*}(\mathcal{K}, \mathcal{A})=(j-1) ! \int_{\mathcal{A}} \operatorname{detr}_{j-1}(A(z)) \mathcal{H}_{K-1}(d x) .
$$

Proof. Letting $\eta_{x}$ be the unique outward pointing normal vector at $x$ on $\partial \mathcal{K}$, observe that for a 
patch $\mathcal{A}$ on $\partial \mathcal{K}$

$$
\begin{aligned}
& \mathcal{H}_{K-1}\left(\mathcal{A}_{\epsilon}\right)=\mathcal{H}_{K-1}\left(\left\{x+\epsilon \frac{\Theta \eta_{x}}{\left\|\eta_{x}\right\|_{\Theta}}: x \in \mathcal{A}\right\}\right) \\
= & \int_{\mathcal{A}} \operatorname{det}\left(I_{K-1}+\epsilon \cdot A(z)\right) \mathcal{H}_{K-1}(d x) \\
= & \sum_{j=1}^{K} \epsilon^{j-1} \int_{\mathcal{A}} \operatorname{detr}_{j-1}(A(z)) \mathcal{H}_{K-1}(d x) \\
= & \sum_{j=1}^{K} \frac{\epsilon^{j-1}}{(j-1) !} \mathcal{M}_{j}^{*}(\mathcal{K}, \mathcal{A})
\end{aligned}
$$

with

$$
\mathcal{M}_{j}^{*}(\mathcal{K}, \mathcal{A})=(j-1) ! \int_{\mathcal{A}} \operatorname{detr}_{j-1}(A(z)) \mathcal{H}_{K-1}(d x) .
$$

The first equality follows from 3.1, while the third one follows using the change of basis matrix transformation derived in 3.3 .

We finally use the Taylor Series expansion of the integral of function

$$
\varphi=\frac{1}{(2 \pi)^{K / 2}} e^{-\|x\|^{2}}
$$

over $T^{D}(\mathcal{K}, \epsilon)$ and 3.4 to get an expansion of Gaussian volume of $T^{D}(\mathcal{K}, \epsilon)=\mathcal{K} \oplus B_{D, \mathbb{R}^{K}}(\epsilon)$, which gives the main theorem in this section.

Theorem 3.5. Under the condition

$$
\int_{\partial \mathcal{K}} \frac{1}{1+\|z\|^{\beta}} \mathcal{M}_{j}^{*}(\mathcal{K}, d z) \text { being bounded for all } 0 \leq j \leq K, \text { for some } \beta>0
$$

the Gaussian volume of $T^{\Theta}(\mathcal{K}, \epsilon)$, denoted by $\gamma^{\mathbb{R}^{K}}\left(T^{\Theta}(\mathcal{K}, \epsilon)\right)$, can be represented as the following expansion

$$
\gamma^{\mathbb{R}^{K}}\left(T^{\Theta}(\mathcal{K}, \epsilon)\right)=\mathcal{M}_{0}^{\gamma_{\mathbb{R} K}, D}(\mathcal{K})+\sum_{l=1}^{n+K} \frac{\epsilon^{l}}{l !} \mathcal{M}_{l}^{\gamma_{\mathbb{R}}, D}(\mathcal{K})+\mathcal{R}(\mathcal{K})
$$

with the remainder term in the expansion $\mathcal{R}(\mathcal{K})$ is bounded above as

$$
\mathcal{R}(\mathcal{K}) \leq \frac{\epsilon^{n+2}}{(n+2) !} C(\mathcal{K})
$$


$C(\mathcal{K})$ being a constant. The coefficients in the expansion are given by

$$
\mathcal{M}_{0}^{\gamma_{\mathbb{R} K}, D}(\mathcal{K})=\int_{\mathcal{K}} \varphi(x) d \lambda_{\mathbb{R}^{K}}
$$

and for $l \geq 1$,

$$
\begin{aligned}
\mathcal{M}_{l}^{\gamma_{\mathbb{R}^{K}, D}}(\mathcal{K}) & =\sum_{m=0}^{l-1}\left(\begin{array}{c}
l-1 \\
m
\end{array}\right)(-1)^{l-1-m} \int_{\partial \mathcal{K}} \frac{\left\|\Theta^{1 / 2} \eta_{x}\right\|}{\left\|\eta_{x}\right\|} \times\left(\frac{\left\|\Theta \eta_{x}\right\|}{\left\|\Theta^{1 / 2} \eta_{x}\right\|}\right)^{l-1-m} \\
& \times H_{l-1-m}\left(\left\langle x, \frac{\Theta \eta_{x}}{\left\|\Theta \eta_{x}\right\|}\right\rangle\right) \varphi(x) \mathcal{M}_{m+1}^{*}(\mathcal{K}, d x)
\end{aligned}
$$

with $\mathcal{M}_{j}^{*}(\mathcal{K}, d x)$ defined in 3.4 and $\mathcal{M}_{j}^{*}(\mathcal{K},)=$.0 for $j>K$.

Proof. The integral of $\varphi$ over $T^{\Theta}(\mathcal{K}, \epsilon)$ can be obtained by an application of co-area formula (see equation (7.4.14) of Adler and Taylor (2007)). Notice that

$$
T^{\Theta}(\mathcal{K}, \epsilon)=\cup_{0 \leq \delta \leq \epsilon} \partial T^{\Theta}(\mathcal{K}, \delta),
$$

where the foliations $\partial T^{\Theta}(\mathcal{K}, \delta)$ can be considered as level sets of the distance function $V_{\mathcal{K}}$, defined in equation (10), which corresponds to geodesic lengths w.r.t. the Riemannian metric given by

$$
\widetilde{g_{i j}}=\lambda_{i}^{-1} \delta_{i j}
$$

Using Lemma L4 of Bhattacharya et al. (2013) we note that

$$
\nabla V_{\mathcal{K}}=\frac{\Theta^{-1} z}{\left\|\Theta^{-1 / 2} z\right\|}
$$

where $z$ is the vector connecting two points between which the distance is measured. In this setting, as pointed in Lemma 3.1,

$$
z=\frac{\Theta \eta_{x}}{\left\|\Theta^{1 / 2} \eta_{x}\right\|}
$$

where

$$
x=\arg \min _{z \in \mathcal{K}}\|z-y\|^{2},
$$

for $y$ on $\partial T^{\Theta}(\mathcal{K}, \delta)$. Collating equations (18) and (19), and using equation (7.4.14) of Adler and Taylor (2007), we obtain the integral of $\varphi$ over $T^{\Theta}(\mathcal{K}, \epsilon)$ given by

$$
\int_{T^{\Theta}(\mathcal{K}, \epsilon)} \varphi(y) d y=\int_{\mathcal{K}} \varphi(y) d \lambda_{\mathbb{R}^{K}}+\int_{0}^{\epsilon} \int_{\partial \mathcal{K}} \int_{A_{\delta}} \frac{\left\|\Theta^{1 / 2} \eta_{w(y)}\right\|}{\left\|\eta_{w(y)}\right\|} \varphi(y) d \mathcal{H}_{K-1}(y) d \epsilon
$$


where

$$
w(y)=\arg \min _{z \in \mathcal{K}}\|z-y\|^{2} .
$$

We begin our calculation by computing the surface measure of an infinitesimally small patch $\mathcal{A}_{\delta}$ on $\partial T^{D}(\mathcal{K}, \delta)$. Using the parametrization in 3.1

$$
S(N(\mathcal{K})) \ni\left(x, \eta_{x}\right) \mapsto x+\delta \varsigma(x), \text { where } \varsigma(x)=\frac{\Theta \eta_{x}}{\left\|\Theta^{1 / 2} \eta_{x}\right\|}
$$

Note that for $y=x+\delta \varsigma(x)$, we have $w(y)=x$. We invoke a change of variable argument, followed with a Taylor series expansion to obtain:

$$
\begin{aligned}
& \int_{\mathcal{A}_{\delta}} \frac{\left\|\Theta^{1 / 2} \eta_{w(y)}\right\|}{\left\|\eta_{w(y)}\right\|} \varphi(y) d \mathcal{H}_{K-1}(y) \\
= & \sum_{t=0}^{K-1} \int_{\mathcal{A}} \frac{\left\|\Theta^{1 / 2} \eta_{x}\right\|}{\left\|\eta_{x}\right\|} \varphi(x+\delta \varsigma(x)) \frac{\delta^{l}}{l !} \mathcal{M}_{l+1}^{*}(\mathcal{K}, d x) \\
= & \sum_{t=0}^{K-1} \frac{\delta^{l}}{l !} \int_{\mathcal{A}} \frac{\left\|\Theta^{1 / 2} \eta_{x}\right\|}{\left\|\eta_{x}\right\|}\left(\left.\sum_{j=0}^{n} \frac{\delta^{j}}{j !} \frac{\partial^{j} \varphi}{\partial \varsigma^{j}}\right|_{x}+\left.\frac{\delta^{n+1}}{(n+1) !} \frac{\partial^{n+1} \varphi}{\partial \varsigma^{n+1}}\right|_{\alpha(\delta, x)}\right) \mathcal{M}_{l+1}^{*}(\mathcal{K}, d x) \\
= & \sum_{l=0}^{n+K-1} \frac{\delta^{l}}{l !} \sum_{j=0}^{K}\left(\begin{array}{l}
l \\
j
\end{array}\right) \int_{\mathcal{A}} \frac{\left\|\Theta^{1 / 2} \eta_{x}\right\|}{\left\|\eta_{x}\right\|}(-1)^{j}\|\varsigma(x)\|^{j} H_{j}(\langle x, \varsigma(x) /\|\varsigma(x)\|\rangle) \varphi(x) \mathcal{M}_{l-j+1}^{*}(\mathcal{K}, d x) \\
& +\left.\sum_{l=0}^{K-1} \delta^{l} \int_{\mathcal{A}} \frac{\left\|\Theta^{1 / 2} \eta_{x}\right\|}{\left\|\eta_{x}\right\|} \frac{\delta^{n+1}}{(n+1) !} \frac{\partial^{n+1} \varphi}{\partial \varsigma^{n+1}}\right|_{\alpha(\delta, x)} \mathcal{M}_{l+1}^{*}(\mathcal{K}, d x)
\end{aligned}
$$

We see that the remainder in the expansion given by

$$
\mathcal{R}(\mathcal{K})=\left.\sum_{l=0}^{K-1} \delta^{l} \int_{\mathcal{A}} \frac{\left\|\Theta^{1 / 2} \eta_{x}\right\|}{\left\|\eta_{x}\right\|} \frac{\delta^{n+1}}{(n+1) !} \frac{\partial^{n+1} \varphi}{\partial \varsigma^{n+1}}\right|_{\alpha(\delta, x)} \mathcal{M}_{l+1}^{*}(\mathcal{K}, d x)
$$

is indeed bounded above by a constant times

$$
\frac{\delta^{n+1}}{(n+1) !} \lambda_{\Theta}^{\max } \max _{1 \leq i \leq K} \sup _{z}\left(1+\|z\|^{\beta}\right) \frac{\partial^{n+1} \varphi}{\partial z_{i}^{n+1}} \mid \sum_{z}^{K-1} \delta_{l=0}^{l} \int_{\mathcal{A}} \frac{1}{1+\|\alpha(\delta, x)\|^{\beta}} \mathcal{M}_{l+1}^{*}(\mathcal{K}, d x)
$$

where $\lambda_{\Theta}^{\max }$ is the largest eigen value of $\Theta$. Under our assumption (14) and the observation that $\varphi(x)$ has derivatives decaying to 0 faster than inverse powers of $x$, the remainder can be bounded above by constant $C(\mathcal{K})$.

Finally, ignoring remainder and integrating over $\partial \mathcal{K}$ and over $[0, \epsilon]$, the Gaussian volume of 
$T^{D}(\mathcal{K}, \epsilon)$ can be approximated as

$$
\begin{aligned}
& \int_{\mathcal{K}} \varphi d \lambda_{\mathbb{R}^{K}}+\sum_{l=1}^{n+K} \frac{\epsilon^{l}}{l !} \sum_{m=0}^{l-1}\left(\begin{array}{c}
l-1 \\
m
\end{array}\right)(-1)^{l-1-m} \int_{\partial \mathcal{K}} \frac{\left\|\Theta^{1 / 2} \eta_{x}\right\|}{\|\eta(x)\|}\|\varsigma(x)\|^{l-1-m} \\
& \times H_{l-1-m}\left(\left\langle x, \frac{\Theta \eta_{x}}{\left\|\Theta \eta_{x}\right\|}\right\rangle\right) \varphi(x) \mathcal{M}_{m+1}^{*}(\mathcal{K}, d x)
\end{aligned}
$$

which proves (15) with coefficients given by (17).

\section{Expected Euler characteristic}

In this section, we compute the expected Euler characteristic

$$
\mathbb{E}\left[\chi\left(M \cap f^{-1}[u, \infty)\right],\right.
$$

when $y=\left(y_{1}, \ldots, y_{K}\right): M \rightarrow \mathbb{R}^{K}$, where $y_{1}, . ., y_{K}$ satisfy regularity and marginal stationarity with the corresponding gradient field satisfying separability and metric conformity and $M$ is a $C^{3}$ manifold. Similar calculations for the expected Euler characteristic can be found in Adler (1981); Taylor and Adler (2003); Adler and Taylor (2007) with motivation to approximate probabilities of exceeding high levels in Adler (2000) and an explicit approximation in Taylor et al. (2005). Again, we show a reduction of computations to calculation of the Lipschitz curvatures $\left\{\mathcal{L}_{j}(M)\right\}_{0 \leq j \leq n}$, with respect to base spatial metric $g$ and EC density functionals $\tilde{\rho}_{n}(F, u)$. This can be viewed as a de-coupling into geometric information about the underlying manifold, captured by LKCs, and information about the distribution of the field, captured by EC densities.

We begin this section by stating two lemmas, that constitute the basic tools used in the calculation of the expected Euler characteristic and subsequently, in computation of the integral representation of EC densities in 5. The derivation of the averaged Euler characteristic in Taylor (2006) hinges on a clever conditioning on $(y, \nabla y)$, that reduces the math to computing the conditional mean and variance of Gaussian random fields and calculating conditional expectation of double forms $\left(y^{*} \nabla^{2} F\right)^{l}$. We re-derive these calculations adapted to the heterogeneity in the distribution of the component Gaussian fields. The following two lemmas, as emphasized, constitute the main tools for the computations to go through for the heterogeneous Gaussian related random fields.

Lemma 4.1. Conditional Mean and Variance Consider a heterogeneous Gaussian related field $f=F \circ y$ where $y=\left(y_{1}, \ldots, y_{K}\right): M \rightarrow \mathbb{R}^{K}$ is such that $y_{i}$ for $i \in\{1,2, . ., K\}$ are unit variance, centered, independent random fields on a $n$ dimensional manifold $M$ with gradient field, $\nabla y$ having separable covariance structure $D \otimes I$ and inducing conformal metrics, as stated in (C) and (D). 
Then, the conditional mean and covariance of $\nabla^{2} f$, conditioned on $(y, \nabla y)$, are given by

$$
\text { Mean: } \mu_{y, \nabla y}=\mathbb{E}\left(\nabla^{2} f \mid y, \nabla y\right)=y^{*} \nabla^{2} F(y)-\langle D \nabla F(y), y\rangle I_{n}
$$

where the $(i, j)$ th element of $\left(y^{*} \nabla^{2} F(y)\right)_{(i, j)}=\sum_{k=1}^{K} \sum_{k^{\prime}=1}^{K} \frac{\partial^{2} F(y)}{\partial y_{k} \partial y_{k^{\prime}}} \frac{\partial y_{k}}{\partial t_{i}} \frac{\partial y_{k^{\prime}}}{\partial t_{j}}$.

$$
\begin{aligned}
& \text { Variance: } \mathbb{E}\left(\left(\nabla^{2} f-\mu_{y, \nabla y}\right)^{2} \mid y, \nabla y\right)=-\langle D \nabla F(y), D \nabla F(y)\rangle I^{2} \\
& -2\left\langle D^{1 / 2} \nabla F(y), D^{1 / 2} \nabla F(y)\right\rangle R
\end{aligned}
$$

where $R$ is the Riemannian curvature tensor w.r.t. metric $g$.

Proof. Let $\left\{E_{i}\right\}_{i=1}^{m}$ be an orthonormal frame field on $M$, then

$$
\begin{aligned}
\nabla^{2} f\left(E_{i}, E_{j}\right) & =\nabla^{2} F\left(y_{*} E_{i}, y_{*} E_{j}\right)+\sum_{k=1}^{K} \nabla^{2} y_{k}\left(E_{i}, E_{j}\right) \partial_{k} F \\
& =\nabla^{2} F\left(y_{*} E_{i}, y_{*} E_{j}\right)+\left\langle\nabla F(y), \nabla^{2} \underline{y}\left(E_{i}, E_{j}\right)\right\rangle
\end{aligned}
$$

where $\nabla^{2} \underline{y}\left(E_{i}, E_{j}\right)$ represents the vector

$$
\left(\nabla^{2} y_{1}\left(E_{i}, E_{j}\right), \ldots, \nabla^{2} y_{K}\left(E_{i}, E_{j}\right)\right)
$$

\section{Mean:}

$$
\mathbb{E}\left(\nabla^{2} f\left(E_{i}, E_{j}\right) \mid y, \nabla y\right)=\nabla^{2} F\left(y_{*} E_{i}, y_{*} E_{j}\right)+\mathbb{E}\left(\left\langle\nabla F(y), \nabla^{2} \underline{y}\left(E_{i}, E_{j}\right)\right\rangle \mid y, \nabla y\right)
$$

Denoting $\Sigma_{y, \nabla y}$ as the covariance of $(y, \nabla y)$, where $\Sigma_{y, \nabla y}$ has a block diagonal form given as

$$
\Sigma_{y, \nabla y}=\left[\begin{array}{cccc}
I_{K} & 0 & \ldots & 0 \\
0 & \lambda_{1} I_{n} & \ldots & 0 \\
0 & 0 & \ldots & 0 \\
0 & 0 & \ldots & \lambda_{k} I_{n}
\end{array}\right]
$$

and observing that the covariance between $\nabla^{2} \underline{y}\left(E_{i}, E_{j}\right)$ and $(y, \nabla y)$ can be expressed as

$$
\Sigma_{\nabla^{2} \underline{y}\left(E_{i}, E_{j}\right),(y, \nabla y)}=\left[\begin{array}{cccc|c}
-\delta_{i j} \lambda_{1} & 0 & \ldots & 0 & \\
0 & -\delta_{i j} \lambda_{2} & \ldots & 0 & \\
\vdots & \vdots & \ldots & \vdots & \underline{0} \\
0 & 0 & \ldots & -\delta_{i j} \lambda_{K} &
\end{array}\right]=\left[\begin{array}{ll}
-\delta_{i j} D & \underline{0} \\
\frac{0}{\times n K}
\end{array}\right]
$$


we compute the conditional mean of $\nabla^{2} \underline{y}\left(E_{i}, E_{j}\right) \mid y, \nabla y$. Finally, let us denote by $\Sigma_{\nabla^{2} \underline{y}\left(E_{i}, E_{j}\right)}$ the covariance matrix of $\nabla^{2} \underline{y}\left(E_{i}, E_{j}\right)$. With this notation, the conditional mean $\mathbb{E}\left(\nabla^{2} f\left(E_{i}, E_{j}\right) \mid y, \nabla y\right)$ can be expressed as

$$
\begin{aligned}
& \mathbb{E}\left(\nabla^{2} f\left(E_{i}, E_{j}\right) \mid y, \nabla f\right) \\
= & \nabla^{2} F\left(y_{*} E_{i}, y_{*} E_{j}\right)+(\nabla F(y))^{T} \Sigma_{\nabla^{2} \underline{y}}\left(E_{i}, E_{j}\right),(y, \nabla y) \Sigma_{y, \nabla y}^{-1}\left(\begin{array}{c}
y \\
\nabla y
\end{array}\right) \\
= & \nabla^{2} F\left(y_{*} E_{i}, y_{*} E_{j}\right)-\delta_{i j}\left\langle D^{1 / 2} \nabla F(y), D^{1 / 2} y\right\rangle .
\end{aligned}
$$

\section{Variance:}

Remark 4.2. Recall that, for the case of a single Gaussian field with $R(k)$ as Riemannian curvature tensor under the induced metric (Adler and Taylor, 200\%, Lemma 12.2.1)

$$
-2 R(k)=\mathbb{E}\left[\left(\nabla^{2} y_{k}\right)^{2}\right]
$$

which in coordinates can be expressed as

$$
R_{i j m n}(k)=\mathbb{E}\left[\nabla^{2} y_{k}\left(E_{i}, E_{m}\right) \nabla^{2} y_{k}\left(E_{j}, E_{n}\right)-\nabla^{2} y_{k}\left(E_{i}, E_{n}\right) \nabla^{2} y_{k}\left(E_{j}, E_{m}\right)\right]
$$

where,

$$
\nabla^{2} y_{k}\left(E_{i}, E_{m}\right)=\partial_{i m}^{2} y_{k}-\left(\nabla_{\partial_{i}} \partial_{m}\right) y_{k}
$$

Remark 4.3. Note that in case of Gaussian fields with conformity induced by the gradient fields (or the stricter case of isotropic Gaussian random fields), the induced metric is just a constant multiple of the Euclidean metric, where the constant is simply the second spectral moment of the underlying Gaussian random field. In such a case, writing $R(k)$ and $R$ as the Riemannian curvature w.r.t. the induced and the Euclidean metrics respectively, we have

$$
R(k)(X, Y, Z, W)=\lambda_{k} R(X, Y, Z, W)
$$

Noting that

$$
\left[\nabla^{2} f-\mathbb{E}\left(\nabla^{2} f \mid y, \nabla y\right)\right]\left(E_{i}, E_{j}\right)=\left\langle\nabla F(y), \nabla^{2} \underline{y}\left(E_{i}, E_{j}\right)\right\rangle-\delta_{i j}\left\langle D^{1 / 2} \nabla F(y), D^{1 / 2} y\right\rangle
$$

and

$$
\begin{aligned}
& {\left[\nabla^{2} f-\mathbb{E}\left(\nabla^{2} f \mid y, \nabla y\right)\right]^{2}\left(E_{i}, E_{j}, E_{m}, E_{n}\right) } \\
= & {\left[\nabla^{2} f-\mathbb{E}\left(\nabla^{2} f \mid y, \nabla y\right)\right]\left(E_{i}, E_{m}\right)\left[\nabla^{2} f-\mathbb{E}\left(\nabla^{2} f \mid y, \nabla y\right)\right]\left(E_{j}, E_{n}\right) }
\end{aligned}
$$




$$
-\left[\nabla^{2} f-\mathbb{E}\left(\nabla^{2} f \mid y, \nabla y\right)\right]\left(E_{i}, E_{n}\right)\left[\nabla^{2} f-\mathbb{E}\left(\nabla^{2} f \mid y, \nabla y\right)\right]\left(E_{j}, E_{m}\right)
$$

Next conditioning on $(y, \nabla y)$ and taking expectation, it is not difficult to see that

$$
\begin{aligned}
& \mathbb{E}\left(\left[\nabla^{2} f-\mathbb{E}\left(\nabla^{2} f \mid y, \nabla y\right)\right]^{2} \mid y, \nabla y\right)\left(E_{i}, E_{j}, E_{m}, E_{n}\right) \\
= & -\|D \nabla F(y)\|^{2} I^{2}\left(E_{i}, E_{j}, E_{m}, E_{n}\right)-2\left\|D^{1 / 2} \nabla F(y)\right\|^{2} R\left(E_{i}, E_{j}, E_{m}, E_{n}\right)
\end{aligned}
$$

The next lemma is a computational tool to compute the conditional expectation

$$
\mathbb{E}\left[\left(y^{*} \nabla^{2} F\right)^{l} \mid y, \nabla f\right]
$$

which we are left to compute after a tower argument with expectation and plugging in the conditional mean and variance, computed in 4.1.

Lemma 4.4. Let $E$ be an orthonormal frame bundle on $T \mathbb{R}^{K}$, then

$$
\mathbb{E}\left[\left(y^{*} \nabla^{2} F\right)^{l} \mid y, \nabla_{E} f\right]=\operatorname{Tr}^{\nabla F^{\perp}}\left(D \nabla^{2} F_{\mid \nabla F^{\perp}}\right)^{l} I^{l}+\operatorname{Err}_{l}
$$

where $\nabla F^{\perp}(y)$ denotes the vector space generated by the span of vectors in $T_{y} \mathbb{R}^{K}$ which are orthogonal to $\nabla F(y)$, and

$$
\operatorname{Err}_{l}=O\left(\left\|\nabla_{E} f\right\|^{2}\left\|\left(\nabla^{2} F\right)^{l}\right\|_{\otimes^{2 l} \mathbb{R}^{K}}\right)
$$

where the big-O notation has the same interpretation as in Taylor (2006).

Proof. Notice that the conditional expectation in above lemma has the same form as that in Corollary 2.3 of Taylor (2006). However, the inner product on $T_{y} \mathbb{R}^{K}$ needs to be defined appropriately so as to match the statement of the aforementioned corollary from Taylor (2006). More precisely, suppose we take an orthonormal frame $\left\{\bar{E}_{1, x}, \ldots, \bar{E}_{d, x}\right\}$ in the parameter space $M$, we could rewrite the conditional expectation as

$$
\mathbb{E}\left[\left(y^{*} \nabla^{2} F\right)^{l} \mid y, \nabla_{E} f\right]=\mathbb{E}\left[\left(y^{*} \nabla^{2} F\right)^{l} \mid y,\left\langle y_{*} E_{1}, \nabla F\right\rangle, \ldots,\left\langle y_{*} E_{d}, \nabla F\right\rangle\right]
$$

Now consider

$$
X_{i, y(x)}=y_{*}\left(\bar{E}_{i, x}\right)=\left.\sum_{j=1}^{K}\left\langle\nabla y_{j}, E_{i, x}\right\rangle \frac{\partial}{\partial y_{j}}\right|_{y(x)} .
$$

This is Gaussian on $T_{y(x)} \mathbb{R}^{K}$ and as $i$ ranges over $\{1, \ldots, d\}$ we get IID copies, their distribution is 
$\gamma_{V}$ (conditional on $y(x)$ ). However to invoke Corollary 2.3 of Taylor (2006), we shall rewrite

$$
X_{i, y(x)}=\left.\sum_{j=1}^{k}\left(\lambda_{j}^{-1 / 2}\left\langle\nabla y_{j}, E_{i, x}\right\rangle\right) \lambda_{j}^{1 / 2} \frac{\partial}{\partial y_{j}}\right|_{y(x)}
$$

where $\left(\lambda_{j}^{-1 / 2}\left\langle\nabla y_{j}, E_{i, x}\right\rangle\right)$ are i.i.d. standard normal. This, in turn leads us to define a new inner product $\langle\cdot, \cdot\rangle_{D^{-1}}$ on $T_{y(x)} \mathbb{R}^{K}$ as

$$
\langle V, W\rangle_{D^{-1}}=\sum_{i=1}^{K} \lambda_{i}^{-1} V_{i}(x) W_{i}(x)
$$

ensuring that

$$
\left(\left.\lambda_{j}^{1 / 2} \frac{\partial}{\partial y_{j}}\right|_{y(x)}\right), \quad 1 \leq j \leq K
$$

is an orthonormal basis on $T_{y} \mathbb{R}^{K}$ w.r.t. the new inner product. With this notation, we define $\nabla F^{\perp}$ as the linear subspace of $T_{y} \mathbb{R}^{K}$ consisting of vectors orthogonal to $D \nabla F$.

The next theorem computes the conditional expectation of double form $-\left(\nabla^{2} f\right)^{n}$, when conditioned on $y$ and $\nabla f$. This leads to the derivation of the expected Euler Characteristic of excursion set,

$$
M \cap f^{-1}[u, \infty)=\{t \in M: f(t) \geq u\} .
$$

Theorem 4.5. Expected Euler Characteristic: Let heterogeneous Gaussian related field, as considered in 4.1, be denoted by $f=F \circ y$, with component heterogeneous, but independent Gaussian units

$$
y=\left(y_{1}, \ldots, y_{K}\right): M \rightarrow \mathbb{R}^{k} .
$$

(A). For each $t \in M$

$$
\frac{1}{n !} \mathbb{E}\left[\left(-\nabla^{2} f\right)^{n} \mid y, \nabla f\right](t)=\sum_{j=0}^{\left\lfloor\frac{n}{2}\right\rfloor} \frac{\left(-R_{t}\right)^{j}}{j !} \alpha_{j}(t)
$$

where $R$ is the Riemannian curvature tensor of the Manifold wrt to metric $g$ and $\alpha_{j}(t)$ represent random double forms.

(B). The expected Euler Characteristic can be represented as

$$
\mathbb{E} \chi\left(M \cap f^{-1}([u, \infty))=\sum_{j=0}^{n} \mathcal{L}_{j}(M) \rho_{j}(F, u)\right.
$$


for functionals $\rho_{j}(F, u)$ representing the $E C$ densities and $\mathcal{L}_{j}(M)$ representing the $L K C$ measures.

Proof. (A). In the proof, the expectation is evaluated at $t$, but, we choose to suppress the notation $t$ for convenience. To prove the first part of the theorem, we use the tower property of expectation to condition on the field $y$ and gradient $\nabla y$. This reduces computations to the conditional mean and variances of a Gaussian random field, that is

$$
\mathbb{E}\left[-\left(\nabla^{2} f\right)^{n} \mid y, \nabla f\right]=\mathbb{E}\left[\mathbb{E}\left[\left(-\nabla^{2} f\right)^{n} \mid y, \nabla y\right] \mid y, \nabla f\right]
$$

We simplify the inner expectation using a binomial expansion of

$$
\left(-\left(\nabla^{2} f-\mu_{y, \nabla y}\right)-\mu_{y, \nabla y}\right)^{n},
$$

and by plugging in the conditional mean and conditional variance using (20) and (21). This follows from the observation that the expectation of a Gaussian double form simplifies into a binomial expansion of its mean and variance, expressed in the below remark.

Remark 4.6. If $Z$ is a Gaussian double form, then

$$
\mathbb{E}\left(Z^{k}\right)=\sum_{i=0}^{\left\lfloor\frac{k}{2}\right\rfloor} \frac{k !}{(k-2 i) ! i ! 2^{i}} \mu^{k-2 i} \Sigma^{i},
$$

where

$$
\mu=\mathbb{E}(Z) \text { and } \Sigma=\mathbb{E}(Z-\mathbb{E}(Z))^{2} .
$$

The reader can check Taylor and Adler (2003) for a derivation of the above.

This gives us

$$
\begin{aligned}
& \frac{1}{n !} \mathbb{E}\left(\left(-\nabla^{2} f+\mu_{y, \nabla y}-\mu_{y, \nabla y}\right)^{n} \mid y, \nabla y\right) \\
= & \frac{1}{n !}(-1)^{n} \sum_{i=0}^{\left\lfloor\frac{n}{2}\right\rfloor} \frac{n !}{(n-2 i) ! i ! 2^{i}}\left[y^{*} \nabla^{2} F(y)-\langle D \nabla F(y), y\rangle I_{n}\right]^{n-2 i} \\
& \times\left[-\langle D \nabla F(y), D \nabla F(y)\rangle I^{2}-2\left\langle D^{1 / 2} \nabla F(y), D^{1 / 2} \nabla F(y)\right\rangle R\right]^{i} \\
= & (-1)^{n} \frac{1}{n !} \sum_{i=0}^{\left\lfloor\frac{n}{2}\right\rfloor} \frac{n !}{(n-2 i) ! i ! 2^{i}}\left[y^{*} \nabla^{2} F(y)-\langle D \nabla F(y), y\rangle I_{n}\right]^{n-2 i} \\
& \times\left\|D^{1 / 2} \nabla F(y)\right\|^{2 i}\left[-\frac{\|D \nabla F(y)\|^{2}}{\left\|D^{1 / 2} \nabla F(y)\right\|^{2}} I^{2}-2 R\right]^{i}
\end{aligned}
$$




$$
\begin{aligned}
= & \sum_{j=0}^{\left\lfloor\frac{n}{2}\right\rfloor} \sum_{i=j}^{\left\lfloor\frac{n}{2}\right\rfloor}(-1)^{n+i} \frac{1}{(n-2 i) ! i ! 2^{i}} \frac{i !}{(i-j) ! j !}\left[\frac{y^{*} \nabla^{2} F(y)}{\left\|D^{1 / 2} \nabla F(y)\right\|}-\frac{\langle D \nabla F(y), y\rangle}{\left\|D^{1 / 2} \nabla F(y)\right\|} I_{n}\right]^{n-2 i} \\
& \left\|D^{1 / 2} \nabla F(y)\right\|^{n}\left(\frac{\|D \nabla F(y)\|^{2}}{\left\|D^{1 / 2} \nabla F(y)\right\|^{2}} I^{2}\right)^{i-j}(2 R)^{j} \\
= & \sum_{j=0}^{\left\lfloor\frac{n}{2}\right\rfloor} \frac{(-R)^{j}}{j !}\left\|D^{1 / 2} \nabla F(y)\right\|^{n} \sum_{i=j}^{\left\lfloor\frac{n}{2}\right\rfloor}(-1)^{i} \frac{1}{(n-2 i) ! i ! 2^{i}} \frac{i !}{(i-j) ! j !}\left(\frac{\|D \nabla F(y)\|^{2}}{\left\|D^{1 / 2} \nabla F(y)\right\|^{2}} I^{2}\right)^{i-j} \\
& \times \sum_{l=0}^{(n-2 i)} \frac{(n-2 i) !}{l !(n-2 i-l) !}\left(y^{*}\left(-\nabla^{2} F(y) /\left\|D^{1 / 2} \nabla F(y)\right\|\right)\right)^{l}\left(\frac{\langle D \nabla F(y), y\rangle}{\left\|D^{1 / 2} \nabla F(y)\right\|} I_{n}\right)^{n-2 i-l} \\
= & \sum_{j=0}^{\left\lfloor\frac{n}{2}\right\rfloor} \frac{(-R)^{j}}{j !}\left\|D^{1 / 2} \nabla F(y)\right\|^{n} \sum_{l=0}^{n-2 j} \frac{\left(y^{*}\left(-\nabla^{2} F(y) /\left\|D^{1 / 2} \nabla F(y)\right\|\right)\right)^{l}}{l !} \\
& \times \frac{(-1)^{(n-2 j-l)}}{(n-2 j-l) !}\left(\frac{\|D \nabla F(y)\|}{\left\|D^{1 / 2} \nabla F(y)\right\|}\right)^{n-2 j-l} H_{n-2 j-l}\left(\frac{\langle D \nabla F(y), y\rangle}{\|D \nabla F(y)\|}\right) I_{n}^{n-2 j-l}
\end{aligned}
$$

The third equality is obtained by a binomial expansion of

$$
\left[-\frac{\|D \nabla F(y)\|^{2}}{\left\|D^{1 / 2} \nabla F(y)\right\|^{2}} I^{2}-2 R\right]^{i}, \text { for each } i \in\left\{0,1, \ldots,\left\lfloor\frac{n}{2}\right\rfloor\right\}
$$

and an interchange of summations. The subsequent equality follows by another binomial expansion of the expression

$$
\left[\frac{y^{*} \nabla^{2} F(y)}{\left\|D^{1 / 2} \nabla F(y)\right\|}-\frac{\langle D \nabla F(y), y\rangle}{\left\|D^{1 / 2} \nabla F(y)\right\|} I_{n}\right]^{n-2 i}, \text { for each } i \in\left\{j, j+1, \ldots,\left\lfloor\frac{n}{2}\right\rfloor\right\} \text {. }
$$

The final equality follows by another interchange of summations and clubbing of terms to get Hermite polynomials as a function of $\frac{\langle D \nabla F(y), y\rangle}{\|D \nabla F(y)\|}$.

With inner expectation evaluated in (25), we apply Lemma 4.4 to evaluate

$$
\mathbb{E}\left[\left(y^{*} \nabla^{2} F\right)^{l} \mid y, \nabla f\right]
$$

which finally yields

$$
\begin{aligned}
& \frac{1}{n !} \mathbb{E}\left[\left(-\nabla^{2} f\right)^{n} \mid y, \nabla f\right] \\
& =\sum_{j=0}^{\left\lfloor\frac{n}{2}\right\rfloor} \frac{(-R)^{j}}{j !} I_{n}^{n-2 j}\left\|D^{1 / 2} \nabla F(y)\right\|^{n} \times \sum_{l=0}^{n-2 j} \frac{(-1)^{(n-2 j-l)}}{(n-2 j-l) !}\left(\frac{\|D \nabla F(y)\|}{\left\|D^{1 / 2} \nabla F(y)\right\|}\right)^{n-2 j-l}
\end{aligned}
$$




$$
\times H_{n-2 j-l}\left(\frac{\langle D \nabla F(y), y\rangle}{\|D \nabla F(y)\|}\right) \times \operatorname{Tr}^{\nabla F^{\perp}}\left(D \nabla^{2} F_{\mid \nabla F^{\perp}} /\left\|D^{1 / 2} \nabla F(y)\right\|\right)^{l} .
$$

We ignore the error term in 4.4, that contributes

$$
\sum_{j=0}^{\left\lfloor\frac{n}{2}\right\rfloor} \sum_{l=0}^{n-2 j} R^{j} I^{n-2 j-l} H_{n-2 j-l}\left(\frac{\langle D \nabla F(y), y\rangle}{\|D \nabla F(y)\|}\right) \operatorname{Err}_{l}
$$

upto constants. This proves (23), with the random forms

$$
\begin{aligned}
\alpha_{j}(t) & =I_{n}^{n-2 j}\left\|D^{1 / 2} \nabla F(y)\right\|^{n} \times \sum_{l=0}^{n-2 j} \frac{(-1)^{(n-2 j-l)}}{(n-2 j-l) !}\left(\frac{\|D \nabla F(y)\|}{\left\|D^{1 / 2} \nabla F(y)\right\|}\right)^{n-2 j-l} \\
& \times H_{n-2 j-l}\left(\frac{\langle D \nabla F(y), y\rangle}{\|D \nabla F(y)\|}\right) \times \operatorname{Tr}^{\nabla F^{\perp}}\left(D \nabla^{2} F_{\left.\mid \nabla F^{\perp} /\left\|D^{1 / 2} \nabla F(y)\right\|\right)^{l}}\right.
\end{aligned}
$$

(B). The second part of the theorem follows from the expectation metatheorem for counting critical points of $f$ in $M$ above level $u$ and index $k$ and Morse's representation for Euler characteristic, that leads to computation of its expected value. We thus have,

$$
\mathbb{E} \boldsymbol{\chi}\left(M \cap f^{-1}([u, \infty))=\int_{M} \mathbb{E}\left[\mathbb{I}(f \geq u) \operatorname{det}\left(-\nabla^{2} f\right) \mid \nabla f=0\right] V o l_{M, g}\right.
$$

Using the definition of trace so that for any double form $A$, we have

$$
\begin{gathered}
\operatorname{det}(A)=\frac{1}{n !} \operatorname{Tr}\left(A^{n}\right), \\
\mathbb{E} \chi\left(M \cap f^{-1}([u, \infty))\right. \\
=\frac{1}{n !} \int_{M} \mathbb{E}\left[\lim _{\epsilon \rightarrow 0} \mathbb{I}(f \geq u,\|\nabla f\|<\epsilon) \operatorname{Tr}^{M}\left(\left(-\nabla^{2} f\right)^{n}\right) \mid \nabla f\right] V o l_{g} \\
=\frac{1}{n !} \int_{M} \mathbb{E}\left(\lim _{\epsilon \rightarrow 0} \mathbb{I}(f \geq u,\|\nabla f\|<\epsilon) \mathbb{E}\left[\operatorname{Tr}^{M}\left(\left(-\nabla^{2} f\right)^{n}\right) \mid f, \nabla f\right]\right) V o l_{g} \\
=\frac{1}{n !} \int_{M} \mathbb{E}\left(\lim _{\epsilon \rightarrow 0} \mathbb{I}(f \geq u,\|\nabla f\|<\epsilon) \operatorname{Tr}^{M} \mathbb{E}\left[\left\{\mathbb{E}\left(-\nabla^{2} f\right)^{n} \mid y, \nabla y\right\} \mid y, \nabla f\right]\right) V o l_{g} .
\end{gathered}
$$

Using (23), we can conclude that $\mathbb{E} \chi\left(M \cap f^{-1}([u, \infty))\right.$ equals

$$
\frac{1}{n !} \int_{M} \sum_{j=0}^{\left\lfloor\frac{n}{2}\right\rfloor}(2 \pi)^{j} \mathbb{E}\left(\lim _{\epsilon \rightarrow 0} \mathbb{I}(f \geq u,\|\nabla f\|<\epsilon) \alpha_{j}\right) \frac{\operatorname{Tr}^{M}(-R)^{j}}{(2 \pi)^{j} j !} V_{\text {ol }}
$$

where $\operatorname{Tr}^{M}(-R)^{j}$, the geometry from the Riemannian structure induced by $g$ contributes to the LKC and the expectation, computed w.r.t the standard Gaussian density on $\mathbb{R}^{K}$ form the 
EC functionals $\rho$.

Remark 4.7. This is an elucidation on Remark 2.3 in 1. If we have a scaled spatial covariance matrix by a factor of $\nu$, that is the induced metric

$$
g_{i, j}^{k}=\lambda_{k} \times \nu \cdot g_{i, j},
$$

and we carry out computations w.r.t. the canonical spatial metric g, then the scaled versions of conditional mean and variance are

$$
\begin{gathered}
\mu_{y, \nabla y}=\mathbb{E}\left(\nabla^{2} f \mid y, \nabla y\right)=y^{*} \nabla^{2} F(y)-\nu\langle D \nabla F(y), y\rangle I_{n} \\
\mathbb{E}\left(\left(\nabla^{2} f-\mu_{y, \nabla y}\right)^{2} \mid y, \nabla y\right)=-\nu^{2}\langle D \nabla F(y), D \nabla F(y)\rangle I^{2} \\
-2 \nu\left\langle D^{1 / 2} \nabla F(y), D^{1 / 2} \nabla F(y)\right\rangle R .
\end{gathered}
$$

Plugging the scaled versions in the calculations, and noting that $\operatorname{Tr}^{M}$ scales as $\nu^{-n}$ and $V o l_{g^{k}}$ scales as $\nu^{n / 2}$, we obtain the expected Euler characteristic as

$$
\sum_{j=0}^{n} \nu^{j / 2} \mathcal{L}_{j}(M) \rho_{j}(F, u)=\sum_{j=0}^{n} \mathcal{L}_{j}^{\nu}(M) \rho_{j}(F, u),
$$

where $\mathcal{L}^{\nu}$ is the LKC computed w.r.t to the induced spatial metric $\nu \cdot g$.

Remark 4.8. To see the GKF in Taylor (2006) as a special case of 4.5, we let $D=I$ in our computations in 4.5. The difference is that our LKCs are computed w.r.t. the base spatial metric g, whereas the ones in Taylor (2006) are w.r.t the induced spatial metric $\nu \cdot g$. Finally, Remark 4.7 shows that our calculations match as we switch to the induced LKCs.

\section{Integral Representation of EC densities}

In this section, we complete the details of proof of 2.5 , through an integral representation for the EC densities of heterogeneous Gaussian related fields with the Gaussian building blocks, $y$ on $\mathbb{R}^{n}$, satisfying (A), (B), (C) and (D), stated in 2. The integral form of EC densities is seen to match with the coefficients in the volume expansion of the ellipsoidal tubes, introduced in 3.

We begin with a lemma, which evaluates a conditional expectation of functions of the random field restricted to the first $n-1$ coordinates, used in calculation of EC density, denoted as $\rho_{n}(F, u)=$ $\rho_{f, n}(u)$.

We introduce few notations for the section. Denote the gradient of $f$ with respect to the first 
$(n-1)$ coordinates only as

$$
\nabla f_{\mid(n-1)}=\left(\frac{\partial f}{\partial t_{1}}, \frac{\partial f}{\partial t_{2}}, \ldots, \frac{\partial f}{\partial t_{n-1}}\right)
$$

the joint density of $\left(f, \nabla f_{\mid(n-1)}\right)$ at $(u, 0)$ as $\phi_{f, \nabla f_{\mid(n-1)}}(u, 0)$, and the Hessian of $f$ restricted to again the first $(n-1)$ coordinates as $\nabla^{2} f_{\mid(n-1)}$.

Lemma 5.1. With the same set up as 4.5 with a heterogeneous Gaussian related field $f$, we have

$$
\begin{aligned}
& \mathcal{N}_{n}(F, y)=\mathbb{E}\left[\left(\frac{\partial f}{\partial t_{n}}\right)^{+} \operatorname{det}\left(-\nabla^{2} f_{\mid(n-1)}\right) \mid y, \frac{\partial f}{\partial t_{i}}, 1 \leq i \leq(n-1)\right] \\
& =\frac{\left\|D^{1 / 2} \nabla F(y)\right\|^{n}}{(2 \pi)^{1 / 2}} \times \sum_{m=0}^{n-1}(-1)^{(n-1-m)}\left(\begin{array}{c}
n-1 \\
m
\end{array}\right)\left(\frac{\|D \nabla F(y)\|}{\left\|D^{1 / 2} \nabla F(y)\right\|}\right)^{n-1-m} \\
& H_{n-1-m}\left(\frac{\langle D \nabla F(y), y\rangle}{\|D \nabla F(y)\|}\right) \times \operatorname{Tr}^{\nabla F^{\perp}}\left(D \nabla^{2} F_{\mid \nabla F^{\perp}} /\left\|D^{1 / 2} \nabla F(y)\right\|^{m}\right)+\operatorname{Tr}\left(\operatorname{Err}^{n-1}\right),
\end{aligned}
$$

where

$$
\operatorname{Err}^{n-1}=\sum_{m=0}^{n-1} I^{n-1-m} H_{n-1-m}(\langle D \nabla F(y), y\rangle) O\left(\left\|\nabla f_{\mid(n-1)}\right\|^{2}\left\|\nabla^{2} F^{l}\right\|_{\otimes^{2 l} \mathbb{R}^{K}}\right) .
$$

Proof. We can write

$$
\begin{aligned}
& \mathbb{E}\left[\left(\frac{\partial f}{\partial t_{n}}\right)^{+} \operatorname{det}\left(-\nabla^{2} f_{\mid(n-1)}\right) \mid y, \frac{\partial f}{\partial t_{i}}, 1 \leq i \leq(n-1)\right] \\
& =\mathbb{E}\left[\left(\frac{\partial f}{\partial t_{n}}\right)^{+} \mid y, \frac{\partial f}{\partial t_{i}}, 1 \leq i \leq(n-1)\right] \mathbb{E}\left[\operatorname{det}\left(-\nabla^{2} f_{\mid(n-1)}\right) \mid y, \frac{\partial f}{\partial t_{i}}, 1 \leq i \leq n-1\right] \\
& =\frac{\left\|D^{1 / 2} \nabla F(y)\right\|}{(2 \pi)^{1 / 2}} \times\left\|D^{1 / 2} \nabla F(y)\right\|^{n-1} \sum_{m=0}^{n-1}(-1)^{(n-1-m)}\left(\begin{array}{c}
n-1 \\
m
\end{array}\right)\left(\frac{\|D \nabla F(y)\|}{\left\|D^{1 / 2} \nabla F(y)\right\|}\right)^{n-1-m} \\
& \times H_{n-1-m}\left(\frac{\langle D \nabla F(y), y\rangle}{\|D \nabla F(y)\|}\right) \times \operatorname{Tr}^{\nabla F^{\perp}}\left(D \nabla^{2} F_{\mid \nabla F^{\perp}} /\left\|D^{1 / 2} \nabla F(y)\right\|^{m}\right)+\operatorname{Tr}\left(\operatorname{Err}^{n-1}\right),
\end{aligned}
$$

where expectation $\mathbb{E}\left[\operatorname{det}\left(-\nabla^{2} f_{\mid(n-1)}\right) \mid y, \frac{\partial f}{\partial t_{i}}, 1 \leq i \leq n-1\right]$ is already evaluated in (26).

The proof hinges on the observation that

$$
\partial f / \partial t_{n} \perp\left(\left\{\partial f / \partial t_{i}, 1 \leq i \leq n-1\right\} ; \nabla^{2} f_{\mid(n-1)}\right) \mid y .
$$

We conclude the section with the derivation of the EC densities in an integral form, which matches with the coefficients in the Taylor expansion of ellipsoidal tubes. 
Theorem 5.2. Under the same set up as 4.5, that is field $f=F \circ y$ with marginally stationary, zeromean, unit variance, independent Gaussian fields with the gradient field having separable covariance structure and metric conformity, as considered in 4.1, the $E C$ density functionals $\left\{\rho_{n}(F, u), n \in\right.$ $\left.\mathbb{Z}^{+}\right\}$, that appear in the series approximation of the expected Euler Characteristic in (24) can be expressed as

$$
\begin{aligned}
\rho_{n}(F, u)= & \frac{1}{(2 \pi)^{n / 2}} \sum_{m=0}^{n-1}(-1)^{n-1-m}\left(\begin{array}{c}
n-1 \\
m
\end{array}\right) \int_{F^{-1}(z)} \frac{\left\|D^{1 / 2} \nabla F(y)\right\|}{\|\nabla F(y)\|}\left(\frac{\|D \nabla F(y)\|}{\left\|D^{1 / 2} \nabla F(y)\right\|}\right)^{n-1-m} \\
& \times H_{n-1-m}\left(\frac{\langle D \nabla F(y), y\rangle}{\|D \nabla F(y)\|}\right) \operatorname{Tr}^{\nabla F^{\perp}}\left(D \nabla^{2} F_{\mid \nabla F^{\perp}} /\left\|D^{1 / 2} \nabla F(y)\right\|\right)^{m} \\
& \times(2 \pi)^{-K / 2} e^{-\|x\|^{2} / 2} d \mathcal{H}_{K-1}(x) .
\end{aligned}
$$

Proof. The EC densities are calculated as

$$
\begin{aligned}
& \rho_{n}(F, u)=\rho_{f, n}(u) \\
= & \mathbb{E}\left[\left(\frac{\partial f}{\partial t_{n}}\right)^{+} \operatorname{det}\left(-\nabla^{2} f_{\mid(n-1)}\right) \mid f=u, \nabla f_{\mid(n-1)}=0\right] \phi_{f, \nabla f_{\mid(n-1)}}(u, 0) \\
= & \lim _{\epsilon \rightarrow 0} \frac{1}{2 \epsilon \mathcal{V}_{n-1}(\epsilon)} \mathbb{E}\left[\mathbb{I}(|f-u|<\epsilon) \mathbb{I}\left(\left\|\nabla f_{\mid(n-1)}\right\|<\epsilon\right)\left(\frac{\partial f}{\partial t_{n}}\right)^{+} \operatorname{det}\left(-\nabla^{2} f_{\mid(n-1)}\right)\right] \\
= & \lim _{\epsilon \rightarrow 0} \frac{1}{2 \epsilon^{n} \mathcal{V}_{n-1}} \mathbb{E}\left[\mathbb{I}(|f-u|<\epsilon) \mathbb{I}\left(\left\|\nabla f_{\mid(n-1)}\right\|<\epsilon\right) \mathcal{N}_{n}(F, y)\right]
\end{aligned}
$$

with

$$
\mathcal{N}_{n}(F, y)=\mathbb{E}\left[\left(\frac{\partial f}{\partial t_{n}}\right)^{+} \operatorname{det}\left(-\nabla^{2} f_{\mid(n-1)}\right) \mid y, \frac{\partial f}{\partial t_{i}}, 1 \leq i \leq(n-1)\right]
$$

calculated in 5.1 , and

$$
\mathcal{V}_{n}(\epsilon)=\operatorname{Vol}(\mathcal{B}(\epsilon)) \text { and } \mathcal{V}_{n}=\operatorname{Vol}(\mathcal{B}(0,1)) \text { with } \mathcal{B}(0, \epsilon) \subset \mathbb{R}^{n}
$$

Noting that

$$
\lim _{\epsilon \rightarrow 0} \frac{1}{2 \epsilon^{n} \mathcal{V}_{n-1}} \mathbb{E}\left[\mathbb{I}(|f-u|<\epsilon) \mathbb{I}\left(\left\|\nabla f_{\mid(n-1)}\right\|<\epsilon\right) \operatorname{Tr}\left(\operatorname{Err}^{n-1}\right)\right]=0
$$

and by defining

$$
\begin{aligned}
\mathcal{C}_{F}(y)=( & \left.\frac{\|D \nabla F(y)\|}{\left\|D^{1 / 2} \nabla F(y)\right\|}\right)^{n-1-m} H_{n-1-m}\left(\frac{\langle D \nabla F(y), y\rangle}{\|D \nabla F(y)\|}\right) \\
& \times \operatorname{Tr}^{\nabla F^{\perp}}\left(D \nabla^{2} F_{\mid \nabla F^{\perp}} /\left\|D^{1 / 2} \nabla F(y)\right\|\right)^{m},
\end{aligned}
$$


we have

$$
\begin{aligned}
& \rho_{n}(F, u) \\
& =\lim _{\epsilon \rightarrow 0} \frac{1}{2 \epsilon^{n} \mathcal{V}_{n-1}} \times \sum_{m=0}^{n-1}(-1)^{n-1-m}\left(\begin{array}{c}
n-1 \\
m
\end{array}\right) \\
& \times \mathbb{E}\left[\mathbb{E}\left[\mathbb{I}(|f-u|<\epsilon) \mathbb{I}\left(\left\|\nabla f_{\mid(n-1)}\right\|<\epsilon\right) \frac{\left\|D^{1 / 2} \nabla F(y)\right\|^{n}}{(2 \pi)^{1 / 2}} \mathcal{C}_{F}(y) \mid y\right]\right] \\
& =\lim _{\epsilon \rightarrow 0} \frac{1}{2 \epsilon} \times \sum_{m=0}^{n-1}(-1)^{n-1-m}\left(\begin{array}{c}
n-1 \\
m
\end{array}\right) \\
& \times \mathbb{E}\left[\frac{\gamma_{\mathbb{R}^{n-1}}\left(\mathbb{B}\left(0, \epsilon /\left\|D^{1 / 2} \nabla F(y)\right\|\right)\right)}{\mathcal{V}_{n-1}\left(\epsilon /\left\|D^{1 / 2} \nabla F(y)\right\|\right)^{n-1}} \mathbb{I}(|F(y)-u|<\epsilon) \frac{\left\|D^{1 / 2} \nabla F(y)\right\|}{(2 \pi)^{1 / 2}} \mathcal{C}_{F}(y)\right] \\
& =\lim _{\epsilon \rightarrow 0} \frac{1}{2 \epsilon} \times \sum_{m=0}^{n-1}(-1)^{n-1-m}\left(\begin{array}{c}
n-1 \\
m
\end{array}\right) \frac{1}{(2 \pi)^{n / 2}} \\
& \times \mathbb{E}\left[\mathbb{I}(|F(y)-u|<\epsilon)\left\|D^{1 / 2} \nabla F(y)\right\| \mathcal{C}_{F}(y)\right] \\
& =\sum_{m=0}^{n-1}(-1)^{n-1-m}\left(\begin{array}{c}
n-1 \\
m
\end{array}\right) \frac{1}{(2 \pi)^{n / 2}} \lim _{\epsilon \rightarrow 0} \frac{1}{2 \epsilon} \mathbb{E}\left[\mathbb{I}(|F(y)-u|<\epsilon)\left\|D^{1 / 2} \nabla F(y)\right\| \mathcal{C}_{F}(y)\right] \\
& =\sum_{m=0}^{n-1}(-1)^{n-1-m}\left(\begin{array}{c}
n-1 \\
m
\end{array}\right) \frac{1}{(2 \pi)^{n / 2}} \\
& \times \lim _{\epsilon \rightarrow 0} \frac{1}{2 \epsilon} \int_{(u-\epsilon, u+\epsilon)} \int_{F^{-1}(z)} \frac{\left\|D^{1 / 2} \nabla F(y)\right\|}{\|F(y)\|} \mathcal{C}_{F}(y) \frac{1}{(2 \pi)^{K / 2}} e^{-\|y\|^{2} / 2} d \mathcal{H}_{K-1}(y) d z \\
& =\frac{1}{(2 \pi)^{n / 2}} \sum_{m=0}^{n-1}(-1)^{n-1-m}\left(\begin{array}{c}
n-1 \\
m
\end{array}\right) \tilde{\mathcal{C}}_{F}(u)
\end{aligned}
$$

where

$$
\tilde{\mathcal{C}}_{F}(z)=\int_{F^{-1}(z)} \mathcal{C}_{F}(y) \frac{1}{(2 \pi)^{K / 2}} e^{-\|x\|^{2} / 2} d \mathcal{H}_{K-1}(y) .
$$

The proof is complete by applying Federer's co-area formula in the penultimate equality, that is

$$
\begin{aligned}
& \int\left\|D^{1 / 2} \nabla F(y)\right\| \mathbb{I}(|F(y)-u|<\epsilon) \mathcal{C}_{F}(y) \frac{1}{(2 \pi)^{K / 2}} e^{-\|y\|^{2} / 2} d y \\
= & \int \frac{\left\|D^{1 / 2} \nabla F(y)\right\|}{\|\nabla F(y)\|} \mathbb{I}(|F(y)-u|<\epsilon) \mathcal{C}_{F}(y) \frac{1}{(2 \pi)^{K / 2}} e^{-\|y\|^{2} / 2}\|\nabla F(y)\| d y \\
= & \int_{(u-\epsilon, u+\epsilon)} \int_{F^{-1}(z)} \frac{\left\|D^{1 / 2} \nabla F(y)\right\|}{\|F(y)\|} \mathcal{C}_{F}(y) \frac{1}{(2 \pi)^{K / 2}} e^{-\|y\|^{2} / 2} d \mathcal{H}_{K-1}(y) d z .
\end{aligned}
$$


This final theorem helps conclude that the coefficients in the Gaussian volume expansion of an ellipsoidal tube, introduced in 3 do match with the EC densities appearing in the expansion of the expected Euler characteristic. Thus, we complete the details of the proof of 2.5 .

\section{An application}

Let $\left\{T(x), x \in S^{2}\right\}$ denote a Gaussian, zero-mean isotropic spherical random field then it is wellknown from (cf.Marinucci and Peccati (2011)) that the following representation holds in the mean square sense

$$
y(x)=\sum_{\ell m} a_{\ell m} \zeta_{\ell m}(x)=\sum_{\ell} y_{\ell}(x), \quad y_{\ell}(x)=\sum_{m=-\ell}^{\ell} a_{\ell m} \zeta_{\ell m}(x) .
$$

Here $\left\{\zeta_{\ell m}().\right\}$ denotes the family of spherical harmonics, and $\left\{a_{\ell m}\right\}$ the array of random spherical harmonic coefficients, which satisfy

$$
\mathbb{E} a_{\ell m} \bar{a}_{\ell^{\prime} m^{\prime}}=C_{\ell} \delta_{\ell}^{\ell^{\prime}} \delta_{m}^{m^{\prime}}
$$

Further, $\delta_{a}^{b}$ is the Kronecker delta function, and the sequence $\left\{C_{\ell}\right\}$ represents the angular power spectrum of the field.

The random field $y$ can be shown to be almost surely continuous if the $C_{\ell}$ satisfies the assumption $\sum_{\ell \geq L}(2 \ell+1) C_{\ell}=O\left(\log ^{-2} L\right)$. It is worth noting here that the $y_{\ell}$ also represent random eigenfunctions of the spherical Laplacian:

$$
\Delta_{S^{2}} y_{\ell}=-\ell(\ell+1) y_{\ell}, \ell=1,2, \ldots
$$

More often, spherical eigenfunctions emerge naturally from the analysis of the Fourier components of spherical random fields. In such cases, several (nonlinear) functionals of $y_{\ell}$ assume a great practical importance: to mention a couple, the squared norm of $T_{\ell}$ provides an unbiased sample estimate for the angular power spectrum $C_{\ell}$,

$$
\mathbb{E}\left\{\int_{S^{2}} T_{\ell}^{2}(x) d x\right\}=(2 \ell+1) C_{\ell},
$$

while higher-order power lead to estimates of the so-called polyspectra.

In the framework of cosmological data analysis (or, CMB data analysis), a number of papers have searched for deviations of geometric functionals from the expected behaviour under Gaussianity. Here, the so-called Minkowski functionals have been widely used as tools to probe non-Gaussianity of the field $y(x)$, see Matsubara (2010) and the references therein. Many other works have also focussed on local deviations from the Gaussianity assumption, mainly exploiting the properties of integrated higher order moments (polyspectra), see Pietrobon et al. (2008), Rudjord et al. (2010). 
The univariate Gaussian kinematic formula has already found many important applications to the analysis of cosmological data, see for instance Fantaye et al. (2015); Marinucci and Vadlamani (2016) and the references therein for some discussion or Collaboration et al. (2014) and Ade et al. (2015) for applications to real data. The case of multivariate spherical fields will certainly become much more important in the years to come: to mention just a possible application, we recall that most of future CMB experiments will be focussed on so-called polarization data, which can be modeled as isotropic vector-valued Gaussian fields with three components, usually labelled T, E and $\mathrm{B}$ modes in the cosmological literature. $\mathrm{B}$ modes are reckoned to be independent from the $\mathrm{T}$ and E components, so they fall within the framework we developed in this paper.

Consider two spherical, isotropic, Gaussian random fields $y_{1}$ and $y_{2}$ such that they are independent, but not identically distributed, i.e., the the two fields have different angular power spectra $C_{1, \ell}$ and $C_{2, \ell}$, and as before, let the second spectral moment of $y_{1}$ and $y_{2}$ be $\lambda_{1}$ and $\lambda_{2}$, respectively.

Let us consider the nonlinear subordination given by the function $F\left(x_{1}, x_{2}\right)=x_{1}^{2}+x_{2}^{2}$. In the context of Gaussian tube formula, we consider tube around

$$
F^{-1}[u, \infty)=\left\{\left(x_{1}, x_{2}\right) \in \mathbb{R}^{2}: x_{1}^{2}+x_{2}^{2} \geq u\right\}=\mathcal{K}
$$

Since we are considering $M=S^{2}$, therefore, we only are interested in $\mathcal{M}_{l}^{\gamma_{\mathbb{R}^{2}}, D}(\mathcal{K})$ for $l=0$ and $l=2$. The case $l=0$ is simple as it's just the Gaussian volume of $\mathcal{K}$, implying the only nontrivial generalised GMF we are interested in is $\mathcal{M}_{2}^{\gamma_{\mathbb{R}^{2}}, D}(\mathcal{K})$

Using the notation of previous sections,

$$
\begin{aligned}
\mathcal{M}_{2}^{\gamma_{\mathbb{R} K}, D}(\mathcal{K}) & =\sum_{m=0}^{1} \int_{\partial \mathcal{K}}(-1)^{1-m} \times\|\varsigma(x)\|^{1-m} \\
& \times H_{1-m}\left(\left\langle x, \frac{D \eta_{x}}{\left\|D \eta_{x}\right\|}\right\rangle\right) \varphi(x) \mathcal{M}_{m+1}^{*}(\mathcal{K}, d x) \\
& =(-1) \int_{\partial \mathcal{K}}\|\varsigma(x)\| \times H_{1}\left(\left\langle x, \frac{D \eta_{x}}{\left\|D \eta_{x}\right\|}\right\rangle\right) \varphi(x) \mathcal{M}_{1}^{*}(\mathcal{K}, d x) \\
& +\int_{\partial \mathcal{K}} \varphi(x) \mathcal{M}_{2}^{*}(\mathcal{K}, d x)
\end{aligned}
$$

where for $\mathcal{K}$ defined above,

$$
\mathcal{M}_{m+1}^{*}(\mathcal{K}, d x)=m ! \operatorname{detr}_{m}(A(x)) \mathcal{H}(d x),
$$

and $\varsigma(x)=\frac{D \eta_{x}}{\left\|D^{1 / 2} \eta_{x}\right\|}$. Setting $\left(\tilde{E}_{1}, \tilde{E}_{2}\right)$ as the orthonormal basis of $\mathbb{R}^{2}$ with respect to the weighted inner product, with $\tilde{E}_{2}=\varsigma(x)$, and $\tilde{E}_{1}=\frac{E_{1}}{\left\|D^{-1 / 2} E_{1}\right\|}$, where $E_{1}$ is the vector orthogonal to $\eta=\frac{\nabla F}{\|\nabla F\|}$ 
in the usual metric, we observe that $\left(\tilde{E}_{1}, \tilde{E}_{2}\right)$ satisfy $(12)$. With this notation

$$
\begin{aligned}
A(z)_{11} & =\left.\frac{1}{\left\|\eta_{z}\right\|_{\Theta}}\left\langle\nabla_{\tilde{E}_{\theta}} \eta, \tilde{E}_{\theta}\right\rangle_{I}\right|_{z} \\
& =\left.\frac{1}{\|\nabla F(z)\|_{\Theta}}\left\langle\nabla_{\tilde{E}_{1}} \nabla F(z), \tilde{E}_{1}\right\rangle_{I}\right|_{z}
\end{aligned}
$$

Using the standard calculus, and polar coordinates to parametric the set $K$, we obtain

$$
A(u, \theta)_{11}=\frac{1}{u\left(\lambda_{1}^{-1} \cos ^{2} \theta+\lambda_{2}^{-1} \sin ^{2} \theta\right) \sqrt{\lambda_{1} \cos ^{2} \theta+\lambda_{2} \sin ^{2} \theta}}
$$

Therefore, and the above integral in equation (31) reduces to

$$
\begin{aligned}
\mathcal{M}_{2}^{\gamma_{\mathbb{R} K}, D}(\mathcal{K})=(-1) \frac{e^{-u^{2} / 2}}{2 \pi} \int_{\theta \in(0,2 \pi)}\left(\frac{\lambda_{1}^{2} \cos ^{2} \theta+\lambda_{2}^{2} \sin ^{2} \theta}{\lambda_{1} \cos ^{2} \theta+\lambda_{2} \sin ^{2} \theta}\right)^{1 / 2} \\
\quad \times(-1) \frac{\lambda_{1} \cos ^{2} \theta+\lambda_{2} \sin ^{2} \theta}{\sqrt{\lambda_{1}^{2} \cos ^{2} \theta+\lambda_{2}^{2} \sin ^{2} \theta}} d \theta \\
+\frac{e^{-u^{2} / 2}}{2 \pi u} \int_{\theta \in(0,2 \pi)} \frac{1}{\left(\lambda_{1}^{-1} \cos ^{2} \theta+\lambda_{2}^{-1} \sin ^{2} \theta\right) \sqrt{\lambda_{1} \cos ^{2} \theta+\lambda_{2} \sin ^{2} \theta}} d \theta
\end{aligned}
$$

Next, note that $\mathcal{M}_{0}^{\gamma_{\mathbb{R}}{ }^{,} D}(\mathcal{K})=\gamma_{\mathbb{R}^{K}}(\mathcal{K})$, and for the purpose of cosmological applications the parameter space is $S^{2}$, hence $\mathcal{L}_{0}\left(S^{2}\right)=2, \mathcal{L}_{1}\left(S^{2}\right)=0$ and $\mathcal{L}_{2}\left(S^{2}\right)=4 \pi$.

With all this information and equation (8), we can write a precise expression for mean EulerPoincaré characteristic of excursion sets of a random field defined as the sum of squares of two independent, but non-identically distributed Gaussian random fields.

\section{Discussion}

We conclude the paper with few questions that can be addressed in future.

- The expected Euler characteristic draws motivation from being a good approximation to excursion probabilities of the form $\mathbb{P}\left(\sup _{t \in M} f(t) \geq u\right)$ for a wide class of smooth random fields. The statistical implication of approximating such a probability is realized in achieving a control of Family Wise error Rate (FWER) in multiple testing of statistical hypotheses at each $t \in M$. While it is known from Taylor et al. (2005) that the Euler characteristic heuristic holds explicitly for the Gaussian case with exponentially decaying errors, that is

$$
\mid \mathbb{P}\left(\sup _{t \in M} f(t) \geq u\right)-E\left(\chi\left(M \cap f^{-1}[u, \infty)\right) \mid \leq C \exp \left(-\alpha u^{2}\right),\right.
$$


for an explicitly computed constant $\alpha$. It remains to see if such an approximation with explicit rates on the error exists for the above fields in consideration.

- Taylor et al. (2009) provide an elegant geometric proof of the GKF through a Poincaré limit approximation of the canonical isotropic process on a unit sphere in $\mathbb{R}^{l}$. More precisely, a GKF is derived by passing on limits via a Poincaré limit theorem from a KFF for an approximating sequence of smooth $\mathbb{R}^{K}$ valued processes on a unit sphere in $\mathbb{R}^{l}$. The approximating processes are given by

$$
y^{(n)}\left(t, g_{n}\right) \text { identical in distribution to } \pi_{\sqrt{n}, n, k}\left(\sqrt{n} g_{n} t\right)
$$

where $g_{n} \in O(n)$ - the orthogonal group in dimension $n$ and $\pi_{\sqrt{n}, n, k}$ denotes the projection from sphere $S_{\sqrt{n}}\left(\mathbb{R}^{n}\right)$ to $\mathbb{R}^{k}$ for $t \in S\left(\mathbb{R}^{l}\right)$. A question in the case of heterogeneous Gaussian related random fields considered in this work would be if the GKF can be re-derived through a a sequence of limiting processes, thereby enabling a classical (albeit asymptotic) geometric interpretation as in Taylor et al. (2009).

Acknowledgment. Jonathan Taylor acknowledges the support of Air Force Office of Sponsored Research grant 113039. Sreekar Vadlamani acknowledges generous support of the AIRBUS Group Corporate Foundation Chair in Mathematics of Complex Systems. SV is also thankful to Domenico Marinucci for many fruitful discussions in the lead up to this work.

\section{References}

P.A.R. Ade, N Aghanim, Y Akrami, PK Aluri, M Arnaud, M Ashdown, J Aumont, C Baccigalupi, AJ Banday, RB Barreiro, et al. Planck 2015 results. xvi. isotropy and statistics of the cmb. arXiv preprint arXiv:1506.07135, 2015.

R. J. Adler. The Geometry of Random Fields. John Wiley \& Sons, Chichester, 1981.

R. J. Adler. On excursion sets, tube formulas and maxima of random fields. Annals of Applied Probability, pages 1-74, 2000.

R. J. Adler and J. E. Taylor. Random fields and geometry. Springer Monographs in Mathematics. Springer, New York, 2007. ISBN 978-0-387-48112-8.

S. Bhattacharya, R. Ghrist, and V. Kumar. Multi-robot coverage and exploration on riemannian manifolds with boundary. Intl. J. Robotics Res., 33(1):113-137, 2013.

L. Bröcker and M. Kuppe. Integral geometry of tame sets. Geom. Dedicata, 82 (1-3):285-323, 2000. ISSN 0046-5755. doi: 10.1023/A:1005248711077. URL http://dx.doi.org/10.1023/A:1005248711077. 
Planck Collaboration et al. Planck 2013 results. xxiii. isotropy and statistics of the cmb. 2014 .

Y. Fantaye, F. K. Hansen, D. Maino, and D. Marinucci. Cosmological applications of the gaussian kinematic formula. Physical Review D, 91(063501), 2015.

A Gray. Tubes addison wesley. Redwood City, CA, 1990.

M. Kac. On the average number of real roots of a random algebraic equation. Bull. Amer. Math. Soc., 43:314-320, 1943.

D. A. Klain and G.-C. Rota. Introduction to Geometric Probability. Cambridge University Press, Cambridge, 1997.

D. Marinucci and G. Peccati. Random Fields on the Sphere. Representation, Limit Theorem and Cosmological Applications. Cambridge University Press, 2011.

D. Marinucci and S. Vadlamani. High-frequency asymptotics for lipschitzkilling curvatures of excursion sets on the sphere. Ann. Appl. Probab., 26(1):462-506, 2016.

T. Matsubara. Analytic Minkowski functionals of the cosmic microwave background: Second-order non-gaussianity with bispectrum and trispectrum. Physical Review D, 81(8):083505, 2010.

D. Pietrobon, A. Amblard, Cabella P. Balbi, A., A. Cooray, and D. Marinucci. Needlet detection of features in wmap cmb sky and the impact on anisotropies and hemispherical asymmetries. Physical Review D, 78:103504, 2008.

S. O. Rice. Mathematical analysis of random noise. Bell System Tech. J., 24:46-156, 1945.

O. Rudjord, F.K. Hansen, X. Lan, D. Liguori, M. Marinucci, and S. Matarrese. Directional variations of the non-gaussianity parameter $f_{N L}$. Astrophysical Journal, 708:1321-1325, 2010.

L. A. Santaló. Integral geometry and geometric probability. Cambridge Mathematical Library. Cambridge University Press, Cambridge, second edition, 2004. ISBN 0-521-52344-3. doi: 10.1017/ CBO9780511617331. URL http://dx.doi.org/10.1017/CB09780511617331. With a foreword by Mark Kac.

R. Schneider. Convex bodies: the Brunn-Minkowski theory. Number 151. Cambridge University Press, 2013.

R. Schneider and W. Weil. Integralgeometrie. Teubner Skripten zur Math. Stochastik, Stuttgart, 1992.

R. Schneider and W. Weil. Stochastic and Integral Geometry. Springer-Verlag, Berlin Heidelberg, 2008. 
J. Taylor, A. Takemura, and R. J. Adler. Validity of the expected euler characteristic heuristic. Annals of Probability, pages 1362-1396, 2005.

J. E. Taylor. A gaussian kinematic formula. The Annals of Applied Probability, 34(1):122-158, 2006.

J. E. Taylor and R. J. Adler. Euler characteristics for gaussian fields on manifolds. Annals of Probability, pages 533-563, 2003.

J. E. Taylor, R. J. Adler, et al. Gaussian processes, kinematic formulae and poincaré?s limit. The Annals of Probability, 37(4):1459-1482, 2009.

H. Weyl. On the volume of tubes. American Journal of Mathematics, 61(2):461-472, 1939.

K.. J. Worsley. Local maxima and the expected euler characteristic of excursion sets of $\chi 2$, f and t fields. Advances in Applied Probability, pages 13-42, 1994.

K. J. Worsley. Boundary corrections for the expected euler characteristic of excursion sets of random fields, with an application to astrophysics. Advances in Applied Probability, pages 943-959, 1995.

Snigdha Panigrahi, Department of Statistics, Stanford University.

E-mail address, Snigdha Panigrahi: snigdha@stanford.edu

Jonathan Taylor, Department of Statistics, Stanford University.

E-mail address,Jonathan Taylor : jonathan.taylorestanford.edu

Sreekar Vadlamani, TIFR- Center for Applicable Mathematics, Bangalore

E-mail address, Sreekar Vadlamani: sreekar@math.tifrbang.res.in 